\title{
Instability and vibration of multi-member columns subjected to Euler's load
}

Received: 25 May 2015 / Accepted: 22 September 2015 / Published online: 1 October 2015

(C) The Author(s) 2015. This article is published with open access at Springerlink.com

\begin{abstract}
In this paper the instability and vibration phenomena of columns composed of three elements with different bending and compression stiffnesses are presented. In the internal rod the crack is taken into consideration and is being modeled by means of a rotational spring with linear characteristic. The boundary problem has been formulated on the basis of the Hamilton's principle. The proposed general form of problem formulation allows one to create five different systems on the basis of one mathematical model. The final formulation of the boundary problem has been carried out by means of a small parameter method. The monitoring of the columns is done by the analysis of the characteristic curves and shape modes. The obtained results are compared to the ones calculated for the uncracked columns. The results of numerical calculation are concern on vibration frequency, bifurcation load magnitude and bifurcation load-crack size relationship.
\end{abstract}

Keywords Vibration $\cdot$ Instability $\cdot$ Crack $\cdot$ Euler $\cdot$ Column

\section{Introduction}

In the supporting systems such as columns which are classified as slender systems due to much greater length than the cross-sectional area, the monitoring of dynamic behavior is very important. The results of this process are often presented in the plane external load-vibration frequency. The change in the location of the characteristic curve can be observed because of modification of the host structure, the change in the connection stiffness between the elements or by the appearance of cracks. The most dangerous phenomenon in the slender systems is the presence of the crack. That is why the monitoring of the dynamic behavior allows one to predict the crack initiation and/or propagation as well as the type of instability (destruction of the structure).

The scientific papers in which cracks were considered have been written inter alia by Anifantis [2], Binici [6], Chondros [9,10], Chondros and Dimarogonas [11], Chondros et al. [12], Lee and Bergman [15] and Sokół [26]. The results of these studies were presented as the dynamic characteristics of the investigated systems.

The analysis of the scientific papers allows one to see two main methods of creation and simulation of cracks in mathematical models. In the first one the cracks are being called always open while in the second the breathing cracks can be found. In the case when the static deflection is greater than the amplitude of vibration, the crack can be classified as always open or as a one that opens and closes regularly-linear problem. In the second case when the static deflection is small in relation to vibration amplitude, the crack opens and closes in time as vibration amplitude dependent—nonlinear problem.

K. Sokół $(\varangle) \cdot$ S. Uzny

Czestochowa University of Technology, Dabrowskiego 73, 42200 Czestochowa, Poland

E-mail: sokol@imipkm.pcz.pl

S. Uzny

E-mail: uzny@imipkm.pcz.pl 


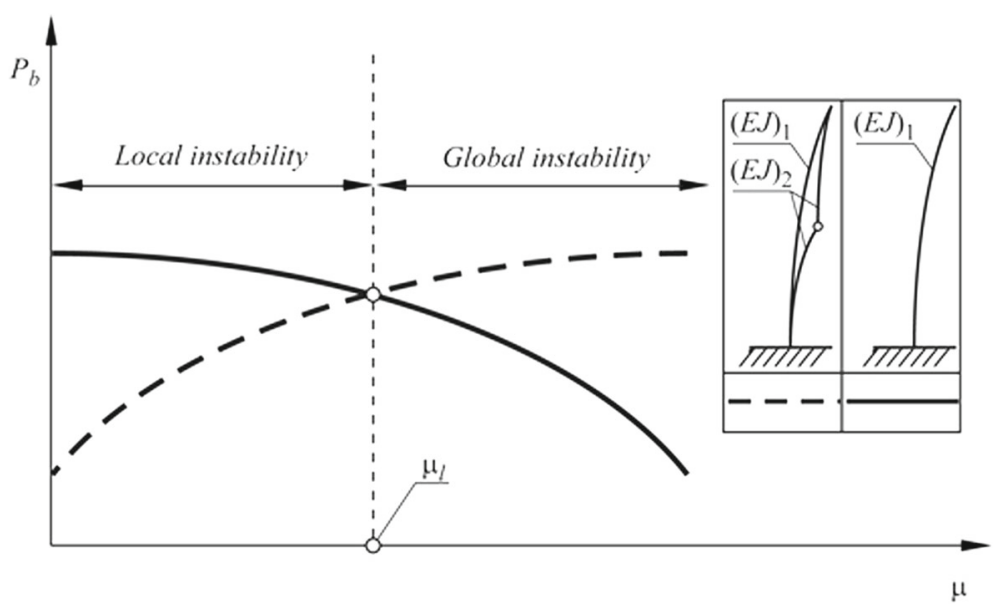

Fig. 1 Regions of local and global instability

On the basis of this division Chondros [10] and Qian [23] have investigated the breathing effect. It can be concluded that if an amplitude is small, the difference in the obtained results for open and breathing cracks is very small. In the case when an amplitude has greater magnitude, this difference is increasing. On the basis of those works the reduced cross-sectional area or massless rotational spring has been proposed as an element which can be used in the crack modeling process.

Peng et al. [22] have proposed crack detection by means of nonlinear output frequency response functions. The nonlinear output frequency response functions (called by authors NOFRFs) were introduced to detect cracks in beams using frequency domain information. On the basis of the analysis of the result it have been concluded that NOFRFs were a sensitive indicator of the presence of cracks. Chati et al. [7] have used the finite element method in order to investigate the edge crack presence in the cantilever beam.

While the slender systems are taken into account, the instability is caused by buckling of the structure. The buckling of such system appears in the perpendicular direction to the axis of the column and smaller moment of inertia. The theoretical and experimental studies were performed by many scientists: Andersen and Thomson [1], Beck [5], Evensen [13], Przybylski [20,21] and Tomski [27]. In the seventies of the previous century Roodr and Chilver [24] have proposed new method of solution of the boundary problems corresponding to slender systems. This method has been presented in detailed form by Osiński [19]. The small parameter method has been used over years by many scientists, and it allows one to present analytical solutions of the systems with rectilinear and curvilinear form of static equilibrium.

The instability of the slender systems can be also presented as a comparison of the nonlinear structure with the linear one. This method allows one to find the local and global instability regions. The local instability of multi-member systems is associated with lower magnitude of bifurcation load in comparison with critical one of the linear structure in which only the rods of higher rigidity are used (see Fig. 1).

The local and global instability phenomenon refers to the deflection from the equilibrium position of the element of the structure which is characterized by a small magnitude of bending and compression stiffnesses (this element deflects as a first one and causes a deflection of higher-stiffness members). The research on this phenomenon has been done by Sokół [25], Tomski and Uzny [28,29] and Tomski and Szmidla [30].

The systems investigated in this paper can be treated as a slender ones due to geometrical features (crosssectional area and the length of elements). Furthermore the method of appliance of the external load and its behavior (the force can change from zero up to maximum magnitude) allow one to simulate the dynamic behavior up to the bifurcation point at which columns are changing the form of equilibrium from the rectilinear into curvilinear.

In this paper the one mathematical model has been proposed in order to simulate crack and different combination of boundary conditions. The crack is being modeled as a massless rotational spring. Stiffness reflects bending moments and the depth of the crack as well as diameter of the cross-sectional area of the cracked element. The natural boundary conditions allow one to satisfy the continuity of longitudinal and transversal displacements, shear forces and bending moments in the point of crack presence. In the scientific papers the authors are focused on one system for which the dedicated method of solution and problem formulation is being presented. In this paper the general formulation has been shown in order to present the solution which 
can be applied to different systems. This generalization is based on discreet elements whose stiffness allows one to simulate different boundary conditions. The investigated complex (multi-member) structure shows the new direction of research into nonlinear slender systems since many scientists have discussed vibrations of beams or single rod columns $[3,4,8,14,17,18]$.

The main scope of this paper is the monitoring of the dynamic behavior of the five columns and comparison of the results of each configuration with the uncracked reference systems. At this point the results of the monitoring are done on the basis of the analysis of the characteristic curves (curves on the plane natural vibration frequency-external load). Leung [16], Sokół [25], Tomski and Uzny [28,29], Tomski and Szmidla [30] and shape modes. The bifurcation load magnitude at which the instability occurs is also presented for each configuration. The results of numerical simulations allow one to predict crack initiation and can be used in the diagnostics of the supporting multi-member slender systems loaded by conservative forces with different combination of boundary conditions. Furthermore the presented generalized form of boundary problem formulation can be easily adapted into more complex system and/or subjected to other loads.

\section{Problem formulation}

The considered in this paper slender supporting system is presented in Fig. 2. It is built out of three elements with different bending and compression stiffnesses. Additionally in the central element the crack appears. Crack is being simulated by means of a rotational spring with linear characteristic. The cracked rod has been divided into two elements (the total length of new elements is equal to the length of the system). The bending and compression stiffness of the rods have been marked as follows $(\mathrm{EJ})_{i},(\mathrm{EA})_{i}$ where $i=1,2,3,4$ and $(\mathrm{EJ})_{1}=(\mathrm{EJ})_{4},(\mathrm{EJ})_{3}=(\mathrm{EJ})_{2}$. The column has been loaded by external compressive Euler's force (the force with constant line of action). In order to present one general problem formulation the discreet elements such as one translational spring and two rotational ones of stiffnesses $C_{\mathrm{T} 1}, C_{\mathrm{R} 0}, C_{\mathrm{R} 1}$, respectively, have been used. In this study the five types of supports were taken into account (see Fig. 2). The individual combination of different types of supports $E j(j=1,2,3,4,5)$ can be achieved by selection of proper springs parameters shown in Fig. 2.

The instability problem has been formulated on the basis of the Hamilton's principle:

$$
\delta \int_{t_{1}}^{t_{2}}\left(\mathrm{E}^{\mathrm{k}}-\mathrm{E}^{\mathrm{p}}\right) \mathrm{d} t=0
$$

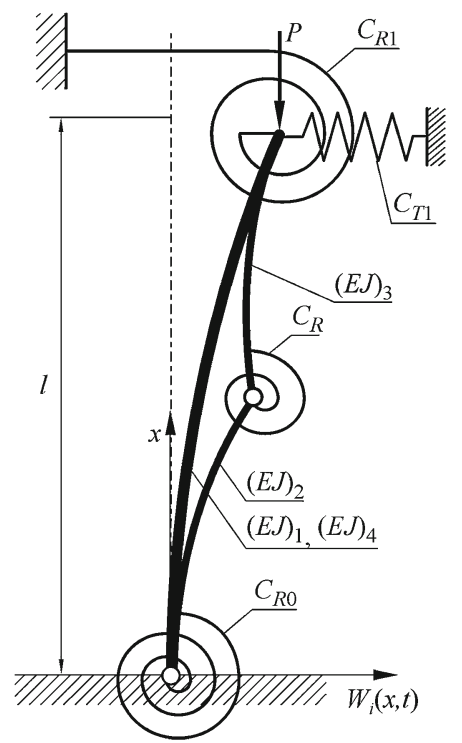

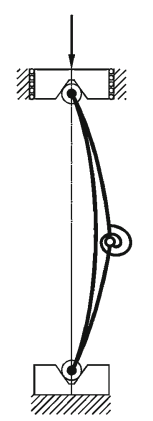

$E_{1}$

$$
C_{R 0}=0
$$

$C_{R 1}=0$

$1 / C_{T 1}=0$

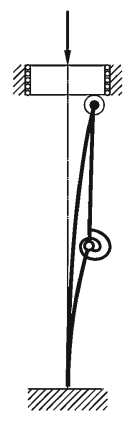

$E_{2}$

$1 / C_{R 0}=0$

$C_{R 1}=0$

$C_{T 1}=0$

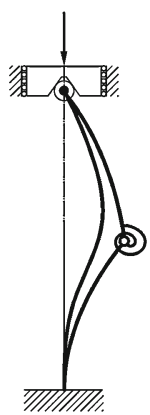

$E_{3}$

$1 / C_{R 0}=0$

$C_{R 1}=0$

$1 / C_{T 1}=0$

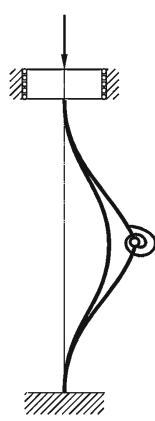

$E_{4}$

$1 / C_{R 0}=0$

$1 / C_{R 1}=0$

$1 / C_{T 1}=0$
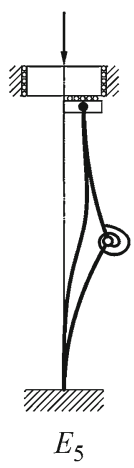

$1 / C_{R 0}=0$

$1 / C_{R 1}=0$

$C_{T 1}=0$

Fig. 2 The considered system with different types of support 
The kinetic energy $E^{\mathrm{k}}$ and potential energy $\mathrm{E}^{\mathrm{p}}$ are expressed as follows :

$$
\begin{aligned}
\mathrm{E}^{\mathrm{k}}= & \frac{1}{2} \sum_{i=1}^{4} \int_{0}^{l_{i}}(\rho A)_{i}\left(\frac{\partial W_{i}\left(x_{i}, t\right)}{\partial t}\right)^{2} \mathrm{~d} x \\
E^{\mathrm{p}}= & \frac{1}{2} \sum_{i=1}^{4}(\mathrm{EJ})_{i} \int_{0}^{l_{i}}\left[\frac{\partial^{2} W_{i}\left(x_{i}, t\right)}{\partial x_{i}^{2}}\right]^{2} \mathrm{~d} x+\frac{1}{2} \sum_{i=1}^{4}(\mathrm{EA})_{i} \int_{0}^{l_{i}}\left[\frac{\partial U_{i}\left(x_{i}, t\right)}{\partial x_{i}}+\frac{1}{2}\left(\frac{\partial W_{i}\left(x_{i}, t\right)}{\partial x_{i}}\right)^{2}\right]^{2} \mathrm{~d} x \\
& +\frac{1}{2} C_{\mathrm{R} 0}\left(\left.\frac{\mathrm{d} W_{i}\left(x_{i}, t\right)}{\mathrm{d} x_{1}}\right|_{x_{1}=0}\right)^{2}+\frac{1}{2} C_{\mathrm{R}}\left(\left.\frac{\mathrm{d} W_{2}\left(x_{2}, t\right)}{\mathrm{d} x_{2}}\right|^{x_{2}=l_{2}}-\left.\frac{\mathrm{d} W_{3}\left(x_{3}, t\right)}{\mathrm{d} x_{3}}\right|_{x_{3}=0}\right)^{2} \\
& +\frac{1}{2} C_{\mathrm{R} 1}\left(\left.\frac{\mathrm{d} W_{1}\left(x_{1}, t\right)}{\mathrm{d} x_{1}}\right|^{x_{1}=l_{1}}\right)^{2}+\frac{1}{2} C_{\mathrm{T} 1}\left[W_{1}\left(l_{1}\right)\right]^{2}+\mathrm{PU}_{1}(l)
\end{aligned}
$$

where $E_{i}$, Young modulus; $J_{i}$, moment of inertia; $A_{i}$, cross-sectional area; $\rho_{i}$, material density; $C_{\mathrm{R}}, C_{\mathrm{R} 0}, C_{\mathrm{R} 1}$, rotational springs stiffness; $C_{\mathrm{T} 1}$, translational spring stiffness; $P$, external load.

Performing variation and integration operations and assuming that virtual displacements $\delta U_{i}\left(x_{i}, t\right)$, $\delta W_{i}\left(x_{i}, t\right)$ for $i=1,2,3,4$ are arbitrary and independent for $0<x_{i}<l_{i}$ allows one to obtain:

- equations of motion $(i=1,2,3,4)$ :

$$
(\mathrm{EJ})_{i} \frac{\partial^{4} W_{i}\left(x_{i}, t\right)}{\partial x_{i}^{4}}-(\mathrm{EA})_{i} \frac{\partial}{\partial x_{i}}\left[\left[\frac{\partial U_{i}\left(x_{i}, t\right)}{\partial x_{i}}+\frac{1}{2}\left(\frac{\partial W_{i}\left(x_{i}, t\right)}{\partial x_{i}}\right)^{2}\right] \frac{\partial W_{i}\left(x_{i}, t\right)}{\partial x_{i}}\right]+(\rho A)_{i} \frac{\partial^{2} W_{i}\left(x_{i}, t\right)}{\partial t^{2}}=0,
$$

- differential equations of longitudinal displacement of the element $(i=1,2,3,4)$ :

$$
\frac{\partial}{\partial x_{i}}\left(\frac{\partial U_{i}\left(x_{i}, t\right)}{\partial x_{i}}+\frac{1}{2}\left[\frac{\partial W_{i}\left(x_{i}, t\right)}{\partial x_{i}}\right]^{2}\right)=0 .
$$

Internal force in each rod is as follows $(i=1,2,3,4)$ :

$$
S_{i}(t)=-(\mathrm{EA})_{i}\left(\frac{\partial U_{i}\left(x_{i}, t\right)}{\partial x_{i}}+\frac{1}{2}\left[\frac{\partial W_{i}\left(x_{i}, t\right)}{\partial x_{i}}\right]^{2}\right)
$$

Introduction of (6) into (4) allows one to express (4) in the form:

$$
(\mathrm{EJ})_{i} \frac{\partial^{4} W_{i}\left(x_{i}, t\right)}{\partial x_{i}^{4}}+S_{i}(t) \frac{\partial^{2} W_{i}\left(x_{i}, t\right)}{\partial x_{i}^{2}}+(\rho A)_{i} \frac{\partial^{2} W_{i}\left(x_{i}, t\right)}{\partial t^{2}}=0
$$

After double integration of (6) the longitudinal displacements in i -th element are as follows:

$$
U_{i}\left(x_{i}, t\right)-U_{i}(0, t)=-\frac{S_{i}(t) x_{i}}{(\mathrm{EA})_{i}}-\frac{1}{2} \int_{0}^{x_{i}}\left[\frac{\partial W_{i}\left(x_{i}, t\right)}{\partial x_{i}}\right]^{2} \mathrm{~d} x_{i} .
$$

The investigated slender system can be described by the following set of geometrical and natural boundary conditions:

$$
W_{1}(0, t)=W_{2}(0, t)=W_{4}(0, t)=0
$$




$$
\begin{aligned}
& \left.\frac{\partial W_{1}\left(x_{1}, t\right)}{\partial x_{1}}\right|_{x_{1}=0}=\left.\frac{\partial W_{2}\left(x_{2}, t\right)}{\partial x_{2}}\right|_{x_{2}=0} \\
& \left.\frac{\partial W_{4}\left(x_{4}, t\right)}{\partial x_{4}}\right|_{x_{4}=0}=\left.\frac{\partial W_{1}\left(x_{1}, t\right)}{\partial x_{1}}\right|_{x_{1}=0} \\
& \left.(\mathrm{EJ})_{1} \frac{\partial^{2} W_{1}\left(x_{1}, t\right)}{\partial x_{1}^{2}}\right|_{x_{1}=0}+\left.(\mathrm{EJ})_{2} \frac{\partial^{2} W_{2}\left(x_{2}, t\right)}{\partial x_{2}^{2}}\right|_{x_{2}=0}+\left.(\mathrm{EJ})_{4} \frac{\partial^{2} W_{4}\left(x_{4}, t\right)}{\partial x_{4}^{2}}\right|_{x_{4}=0} \\
& -\left.C_{\mathrm{R} 0} \frac{\partial W_{1}\left(x_{1}, t\right)}{\partial x_{1}}\right|_{x_{1}=0}=0 \\
& W_{2}\left(l_{2}, t\right)=W_{3}(0, t) \\
& -\left.(\mathrm{EJ})_{2} \frac{\partial^{2} W_{2}\left(x_{2}, t\right)}{\partial x_{2}^{2}}\right|_{x_{2}=l_{2}}+C_{\mathrm{R}}\left[\left.\frac{\partial W_{3}\left(x_{3}, t\right)}{\partial x_{3}}\right|_{x_{3}=0}-\left.\frac{\partial W_{2}\left(x_{2}, t\right)}{\partial x_{2}}\right|_{x_{2}=l_{2}}\right]=0 \\
& \left.(\mathrm{EJ})_{3} \frac{\partial^{2} W_{3}\left(x_{3}, t\right)}{\partial x_{3}^{2}}\right|_{x_{3}=0}+C_{\mathrm{R}}\left[\left.\frac{\partial W_{3}\left(x_{3}, t\right)}{\partial x_{3}}\right|_{x_{3}=0}-\left.\frac{\partial W_{2}\left(x_{2}, t\right)}{\partial x_{2}}\right|_{x_{2}=l_{2}}\right]=0 \\
& \left.(\mathrm{EJ})_{2} \frac{\partial^{3} W_{2}\left(x_{2}, t\right)}{\partial x_{2}^{2}}\right|_{x_{2}=l_{2}}+\left.S_{2} \frac{\partial W_{2}\left(x_{2}, t\right)}{\partial x_{2}}\right|_{x_{2}=l_{2}}-\left.(\mathrm{EJ})_{3} \frac{\partial^{3} W_{3}\left(x_{3}, t\right)}{\partial x_{3}^{2}}\right|_{x_{3}=0} \\
& -\left.S_{3} \frac{\partial W_{3}\left(x_{3}, t\right)}{\partial x_{3}}\right|_{x_{3}=0}=0 \\
& W_{1}\left(l_{1}, t\right)=W_{3}\left(l_{3}, t\right) \\
& W_{4}\left(l_{4}, t\right)=W_{1}\left(l_{1}, t\right) \\
& \left.\frac{\partial W_{1}\left(x_{1}, t\right)}{\partial x_{1}}\right|_{x_{1}=l_{1}}=\left.\frac{\partial W_{3}\left(x_{3}, t\right)}{\partial x_{3}}\right|_{x_{3}=l_{3}} \\
& \left.\frac{\partial W_{4}\left(x_{4}, t\right)}{\partial x_{4}}\right|_{x_{4}=l_{4}}=\left.\frac{\partial W_{1}\left(x_{1}, t\right)}{\partial x_{1}}\right|_{x_{1}=l_{1}} \\
& \left.(\mathrm{EJ})_{1} \frac{\partial^{2} W_{1}\left(x_{1}, t\right)}{\partial x_{1}^{2}}\right|_{x_{1}=l_{1}}+\left.(\mathrm{EJ})_{3} \frac{\partial^{2} W_{3}\left(x_{3}, t\right)}{\partial x_{3}^{2}}\right|_{x_{3}=l_{3}}+\left.(\mathrm{EJ})_{4} \frac{\partial^{2} W_{4}\left(x_{4}, t\right)}{\partial x_{4}^{2}}\right|_{x_{4}=l_{4}} \\
& +\left.C_{\mathrm{R} 1} \frac{\partial W_{1}\left(x_{1}, t\right)}{\partial x_{1}}\right|_{x_{1}=l_{1}}=0 \\
& \left.(\mathrm{EJ})_{1} \frac{\partial^{3} W_{1}\left(x_{1}, t\right)}{\partial x_{1}^{3}}\right|_{x_{1}=l_{1}}+\left.(\mathrm{EJ})_{3} \frac{\partial^{3} W_{3}\left(x_{3}, t\right)}{\partial x_{3}^{3}}\right|_{x_{3}=l_{3}}+\left.(\mathrm{EJ})_{4} \frac{\partial^{3} W_{4}\left(x_{4}, t\right)}{\partial x_{4}^{3}}\right|_{x_{4}=l_{4}} \\
& +C_{\mathrm{T}} W_{1}\left(l_{1}, t\right)+\left.P \frac{\partial W_{1}\left(x_{1}, t\right)}{\partial x_{1}}\right|_{x_{1}=l_{1}}=0 \\
& S_{2}=S_{3} \\
& S_{1}+S_{2}+S_{4}=P \\
& U_{1}(0, t)=U_{2}(0, t)=U_{4}(0, t)=0 \\
& U_{2}\left(l_{2}, t\right)=U_{3}(0, t) \\
& U_{1}\left(l_{1}, t\right)=U_{3}\left(l_{3}, t\right)=U_{4}\left(l_{4}, t\right)
\end{aligned}
$$

The investigations presented in this paper have been done in the non-dimensional form, where

- coordinates and dimensions:

$$
\xi_{i}=\frac{x_{i}}{l_{i}}, \quad d_{i}=\frac{l_{i}}{l_{1}}
$$


- transversal and longitudinal displacements:

$$
w_{i}\left(\xi_{i}, \tau\right)=\frac{W_{i}\left(x_{i}, t\right)}{l_{i}}, \quad u_{i}\left(\xi_{i}, \tau\right)=\frac{U_{i}\left(x_{i}, t\right)}{l_{i}},
$$

- forces:

$$
k_{i}(\tau)=\frac{S_{i}(\tau) l_{i}^{2}}{(\mathrm{EJ})_{i}}, \quad p=\frac{P l_{1}^{2}}{(\mathrm{EJ})_{1}+(\mathrm{EJ})_{2}+(\mathrm{EJ})_{4}},
$$

- vibration frequency:

$$
\omega_{i}^{2}=\Omega^{2} \frac{(\rho A)_{i} l_{i}^{4}}{(\mathrm{EJ})_{i}}, \quad \tau=\Omega t,
$$

- rotational spring stiffness and rigidity ratio:

$$
c=\frac{C_{\mathrm{R}} l_{1}}{(\mathrm{EJ})_{1}+(\mathrm{EJ})_{2}+(\mathrm{EJ})_{4}}, \quad \mu=\frac{(\mathrm{EJ})_{2}}{(\mathrm{EJ})_{1}} .
$$

Due to nonlinearities [integrand expression in Eq. (8)] the small parameter method has been used [28, 29,31]. In this paper only the rectilinear form of static equilibrium is investigated. Additionally the first component of natural vibration frequency is presented which is independent to amplitude of vibration. With so certain assumptions the differential equation of motion in transversal direction after implementation of small parameter method and separation of time and space variables has a form (comp. [28,29,31]):

$$
w_{i}^{I V}\left(\xi_{i}\right)+k_{i 0} w_{i}^{I I}\left(\xi_{i}\right)-\omega_{i}^{2} w_{i}\left(\xi_{i}\right)=0
$$

In (11) $k_{i 0}$ is a non-dimensional longitudinal force which is present in the rods under consideration of rectilinear form of static equilibrium. Distribution of external force onto rods of column is done on the basis of boundary conditions (9r-w) and has a form:

$$
S_{2}=P \frac{(\mathrm{EA})_{2}}{2(\mathrm{EA})_{1}+(\mathrm{EA})_{2}}, \quad S_{1}=\frac{P-S_{2}}{2}, \quad S_{4}=S_{1}
$$

Non-dimensional parameter $\omega_{i 0}$ is as follows:

$$
\omega_{i 0}^{2}=\Omega_{0}^{2} \frac{(\rho A)_{i} l_{i}^{4}}{(\mathrm{EJ})_{i}}
$$

Both $k_{i 0}$ and $\Omega_{0}$ are independent to amplitude of vibration.

The general solution of (11) can be presented in the form $(i=1,2,3,4)$ :

$$
w_{i}\left(\xi_{i}\right)=A_{i} \sin \left(g_{1 i} \xi_{i}\right)+B_{i} \cos \left(g_{1 i} \xi_{i}\right)+C_{i} \sinh \left(g_{2 i} \xi_{i}\right)+D_{i} \cosh \left(g_{2 i} \xi_{i}\right)
$$

where

$$
g_{1 i}=\sqrt{\frac{k_{i 0}}{2}+\sqrt{\frac{k_{i 0}^{2}}{4}+\omega_{i 0}^{2}}}, \quad g_{2 i}=\sqrt{-\frac{k_{i 0}}{2}+\sqrt{{\frac{k_{i 0}}{4}}^{2}+\omega_{i 0}^{2}}} .
$$

Substitution of (14) into boundary conditions allows one to create the system of sixteen homogenous equations with sixteen unknowns (integration constants) $A_{i}, B_{i}, C_{i}, D_{i}(i=1,2,3,4 ; m, n=1,2,3, \ldots, 16)$ :

$$
\left[M_{m n}\right] \operatorname{col}\left\{A_{i}, B_{i}, C_{i}, D_{i}\right\}=0,
$$

Equating the matrix coefficient of zero one obtains the transcendental equation on the basis of which the basic component of vibration frequency at the rectilinear form of static equilibrium is found:

$$
\operatorname{det}\left[M_{m n}\right]=0,
$$

The numerical solution of the determinant (17) leads to results which are describing instability phenomenon as well as the relationship between free vibration frequency and external load. 


\section{Results of numerical simulations}

The third chapter of this paper has been divided into five sub-chapters. In each sub-chapter the results of numerical simulations of one system $E_{j}(j=1,2,3,4,5)$ have been presented. The results include:

- the non-dimensional vibration frequency parameter:

$$
\omega=\sqrt{\Omega_{0}^{2} \frac{\sum_{i=1}^{4}(\rho A)_{i} l_{1}^{4}}{\sum_{i=1}^{4}(\mathrm{EJ})_{i}}}
$$

- external load relationship for different bending rigidity factor magnitude $p$ :

- shape mode and bifurcation load-crack size analysis.

In this paper only the results for central crack location $d_{2}=0.5$ and $(E J)_{1}=(E J)_{4} ;(E J)_{2}=(E J)_{4}$ are being presented because of great number of results for the considered systems in different configurations.

\subsection{Column $\mathrm{E}_{1}$}

In Figs. 3, 4 and 5 the results of investigations on first vibration frequency have been presented for central crack location and different bending rigidity factor $\mu$ taking into account different crack size. The characteristic curves have been plotted in the non-dimensional form. On the axis of external load $\mathrm{p}$ the magnitude of bifurcation force can be found. The bifurcation takes place when the vibration frequency comes to zero. While the very small $(c=100)$ crack is taken into account, the highest loading capacity has been observed. The propagation of the crack up to greater size (curves for $c=1,0.5,0.1,0.0001$ ) causes the reduction in natural vibration frequency of the column as well as loading capacity. The smallest differences in the investigated parameters (bifurcation load and natural vibration frequency) can be found while the crack size changes from $c=100$ down to $c=1$. Regardless of bending rigidity factor $\mu$ magnitude an influence of the crack stays the same-reduction in the loading capacity and vibration frequency. As presented for every $\mu$ the investigated parameters are highly dependent on crack size.

In Fig. 6 an influence of the crack size on bifurcation load for three different magnitudes of bending rigidity factor $(\mu=0.1,0.5,1)$ has been presented. It can be concluded that there is no change in the bifurcation load magnitude for very small crack size. In this case the spring stiffness is above 50 , and it can simulate a

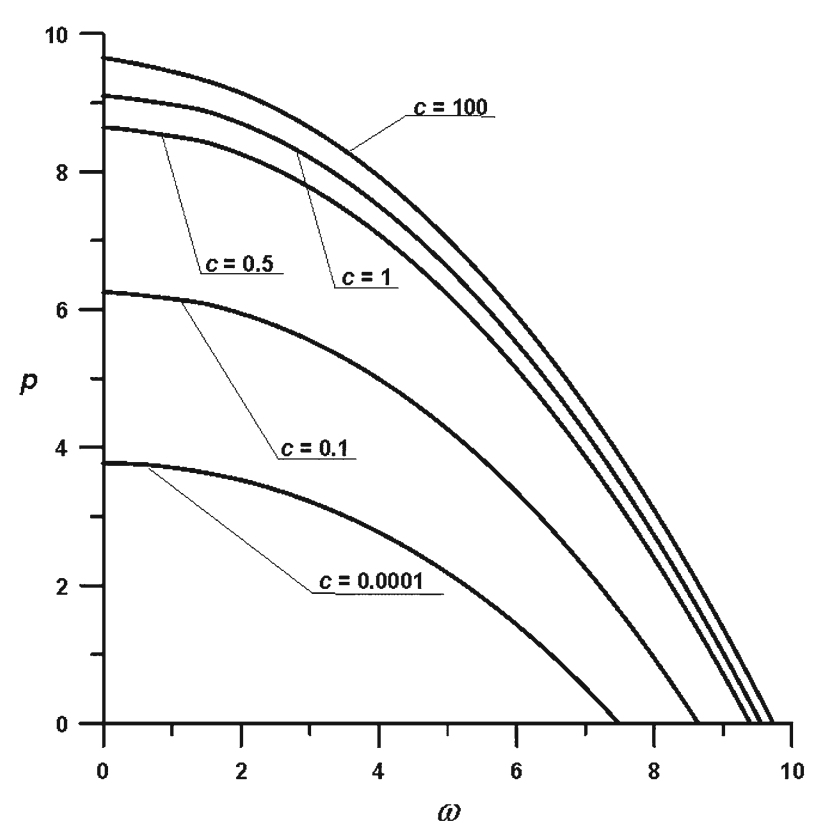

Fig. 3 Characteristic curves on the plane external load-vibration frequency for different crack size, other data: $d_{2}=0.5, \mu=0.1$ 


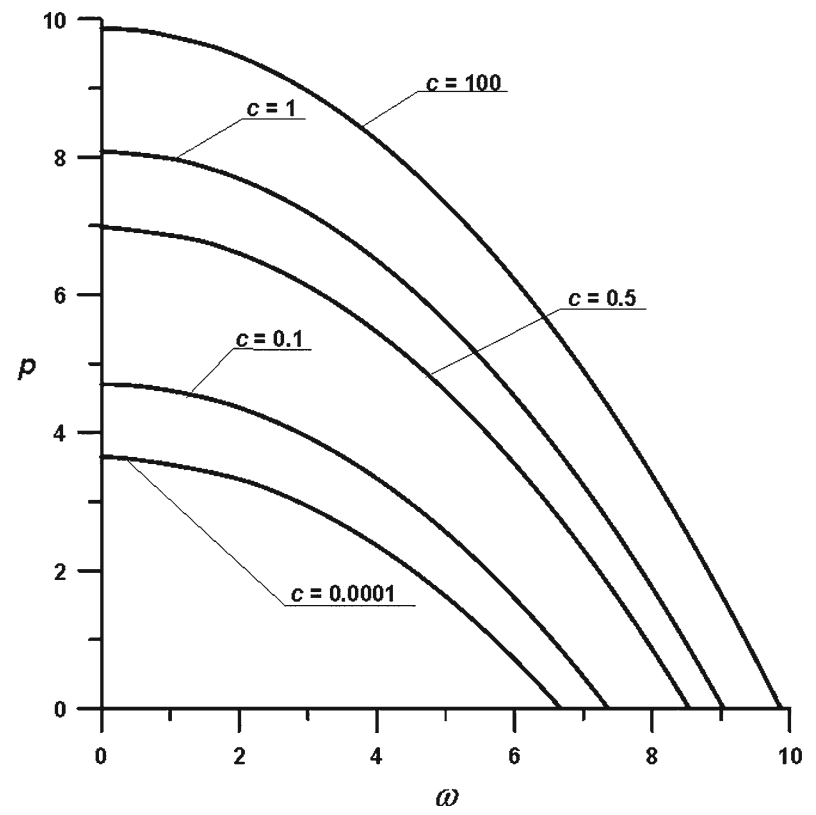

Fig. 4 Characteristic curves on the plane external load-vibration frequency for different crack size, other data: $d_{2}=0.5, \mu=0.5$

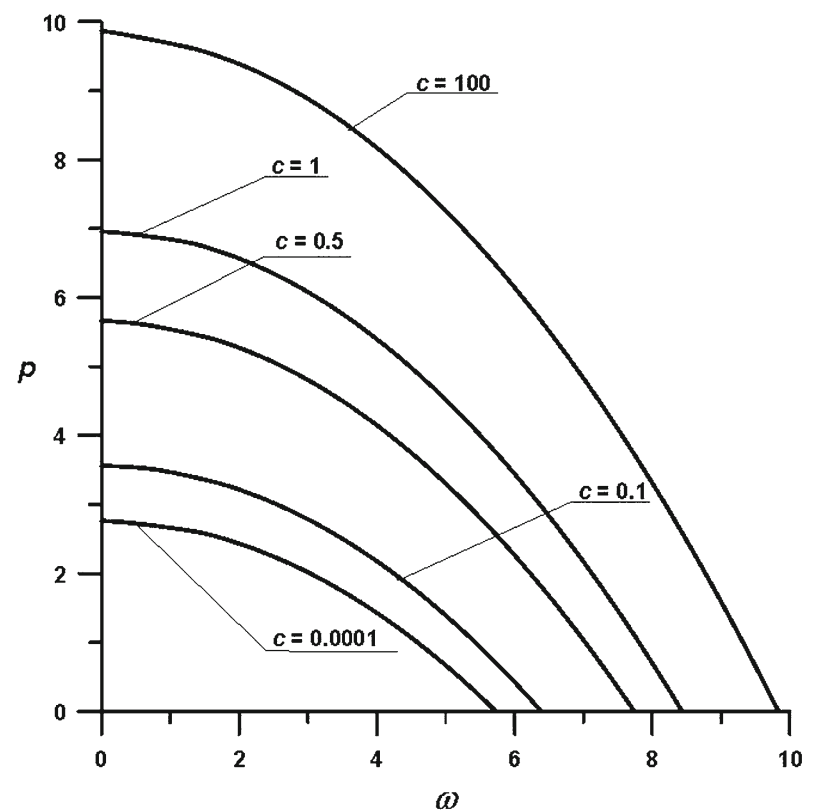

Fig. 5 Characteristic curves on the plane external load-vibration frequency for different crack size, other data: $d_{2}=0.5, \mu=1$

system without crack. As presented in Fig. 6 all the curves regardless of bending rigidity factor magnitude are stabilizing. While the crack size is very big (c close to zero), the rapid decrease in bifurcation load can be observed. For fully cracked member the loading capacity is the lowest.

In Table 1 the first shape modes have been plotted for different crack size. The continuous line corresponds to the external rods ( 1 and 4 ), while the dotted one stands for cracked internal member. The change in bent rods axes shape, especially rods 2 and 3, determines the difference in loading capacity. With an increase in the crack size (reduction in $c$ magnitude), an increase in area between the curves can be observed as well as the good visibility of the crack location (sharp connection between rods). 


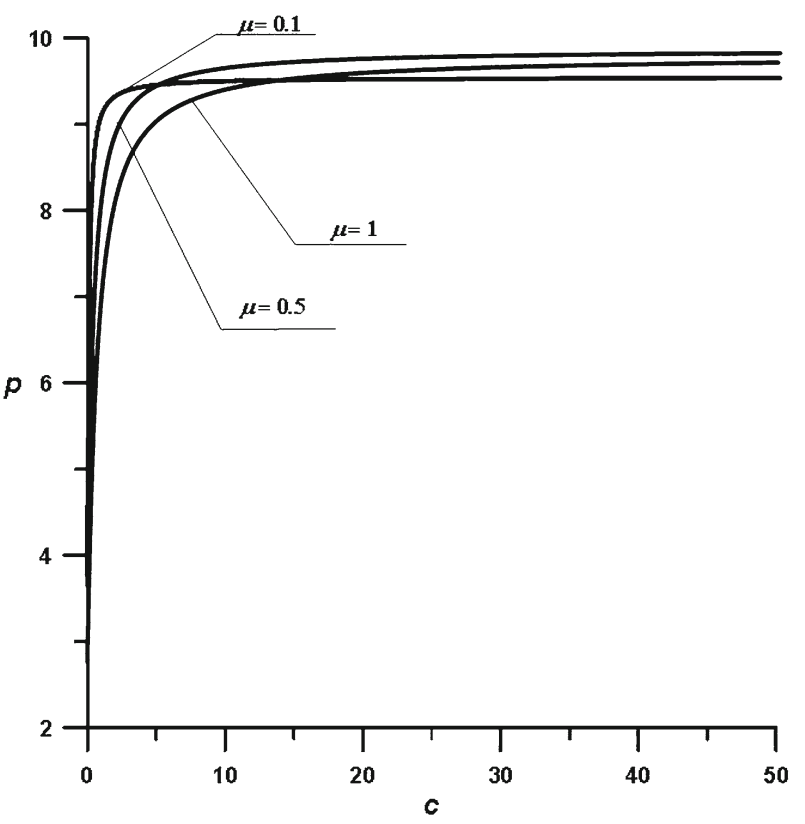

Fig. 6 Curves on the plane external load-crack size for different bending rigidity factor, other data: $d_{2}=0.5$

Table 1 Shape modes, $d_{2}=0.5, \mu=0.1$

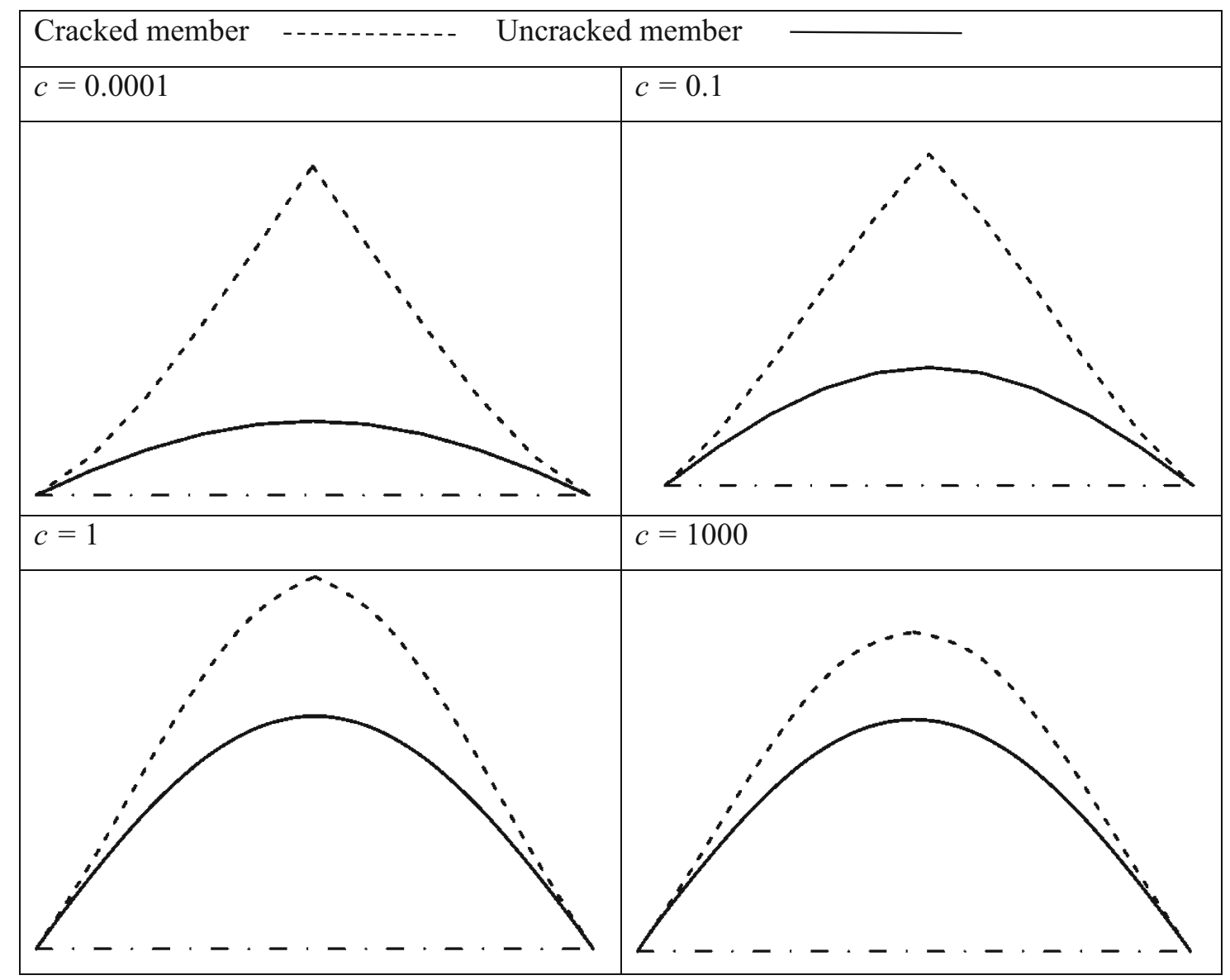




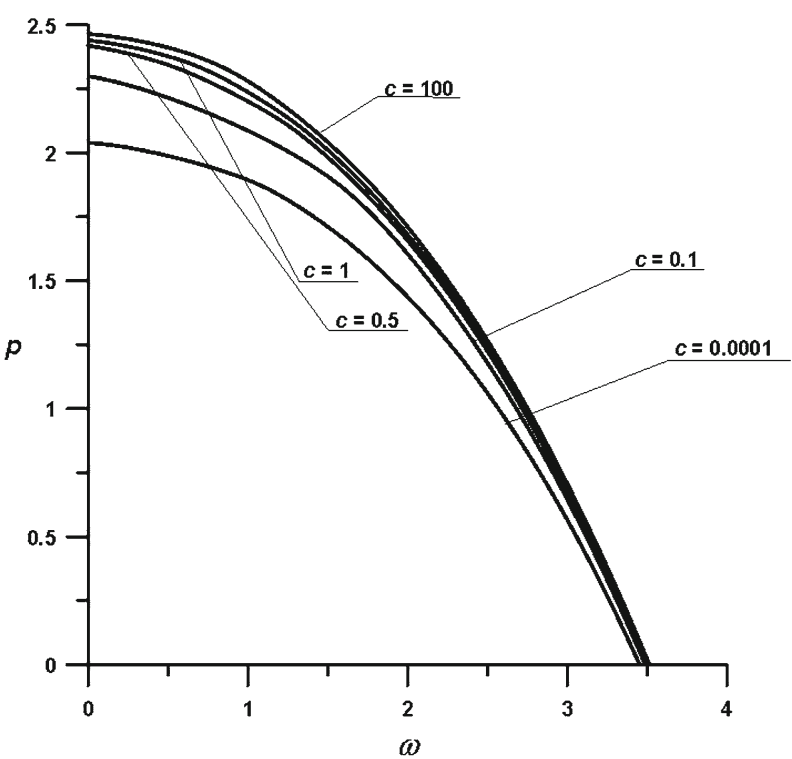

Fig. 7 Characteristic curves on the plane external load-vibration frequency for different crack size, other data: $d_{2}=0.5, \mu=0.1$

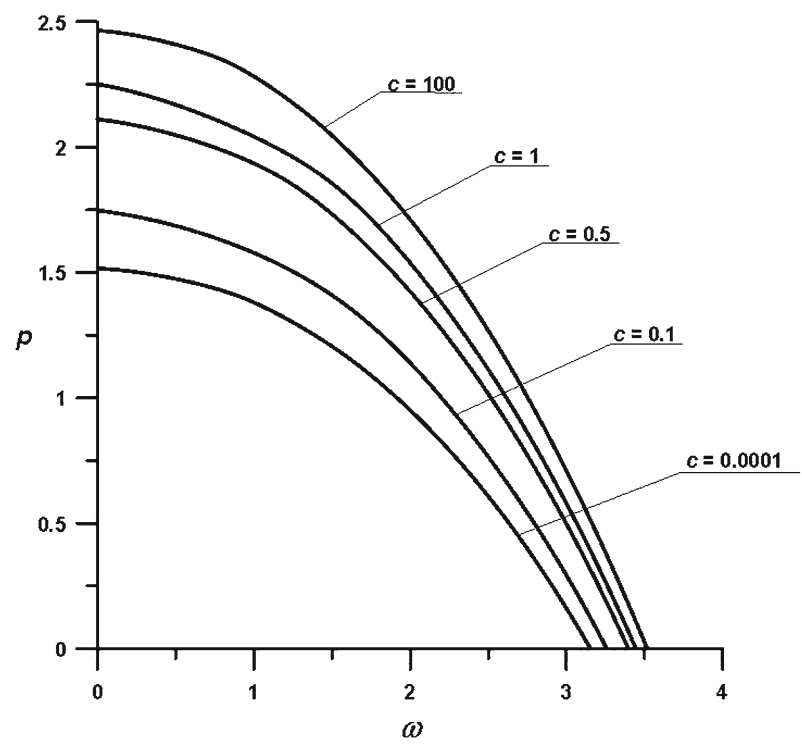

Fig. 8 Characteristic curves on the plane external load-vibration frequency for different crack size, other data: $d_{2}=0.5, \mu=0.5$

\subsection{Column $\mathrm{E}_{2}$}

In Figs. 7, 8 and 9 the results of investigations on first vibration frequency have been presented for central crack location and different bending rigidity factor $\mu$ taking into account different crack size for column in $E_{2}$ configuration. The characteristic curves have been plotted in the non-dimensional form. While the very small $(c=100)$ crack is taken into account, the highest loading capacity has been observed. In the non-dimensional form its magnitude is $\pi^{2} / 4$. This magnitude corresponds to critical loading of the linear cantilever system. An increase in the crack size $(c=1,0.5,0.1,0.0001)$ causes the reduction in natural vibration frequency of the column as well as loading capacity. The smallest differences in the investigated parameters (bifurcation load and natural vibration frequency) can be found while bending rigidity factor is $\mu=0.1$. An increase in $\mu$ translates to greater differences in the position of characteristic curves. For $\mu=1$ the lowest vibration 


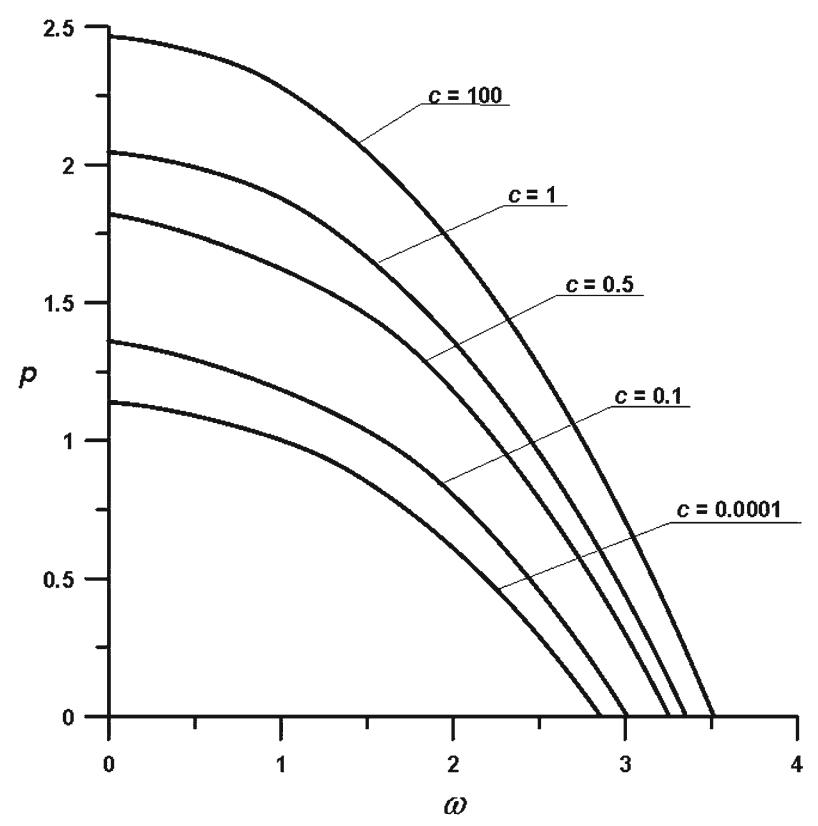

Fig. 9 Characteristic curves on the plane external load-vibration frequency for different crack size, other data: $d_{2}=0.5, \mu=1$

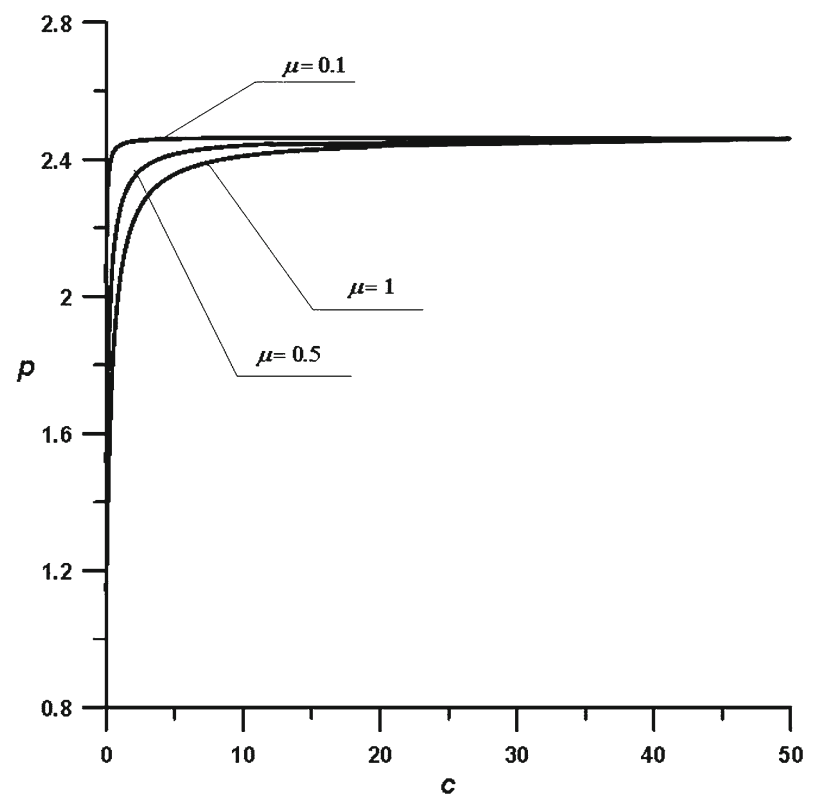

Fig. 10 Curves on the plane external load-crack size for different bending rigidity factor, other data: $d_{2}=0.5$

frequency and loading capacity have been observed. In each configuration the crack causes the reduction in bifurcation load and natural vibration frequency.

In Fig. 10 an influence of the crack size on bifurcation load for three considered magnitudes of bending rigidity factor has been $(\mu=0.1,0.5,1)$ presented. It can be concluded that there is no change in the bifurcation load magnitude for small crack size or this change is insignificant at the beginning. In this case when the spring stiffness is above 50, the system without crack can be simulated. As presented in Fig. 10 all the curves regardless of bending rigidity factor magnitude are stabilizing. While the crack size is very big ( $c$ close to zero), the rapid decrease in bifurcation load can be observed. For $\mu=0.1$ the reduction in spring stiffness causes the most rapid decrease in loading capacity. Fully cracked internal member reflects in the lowest loading capacity. 
Table 2 Shape modes, $d_{2}=0.5, \mu=0.1$

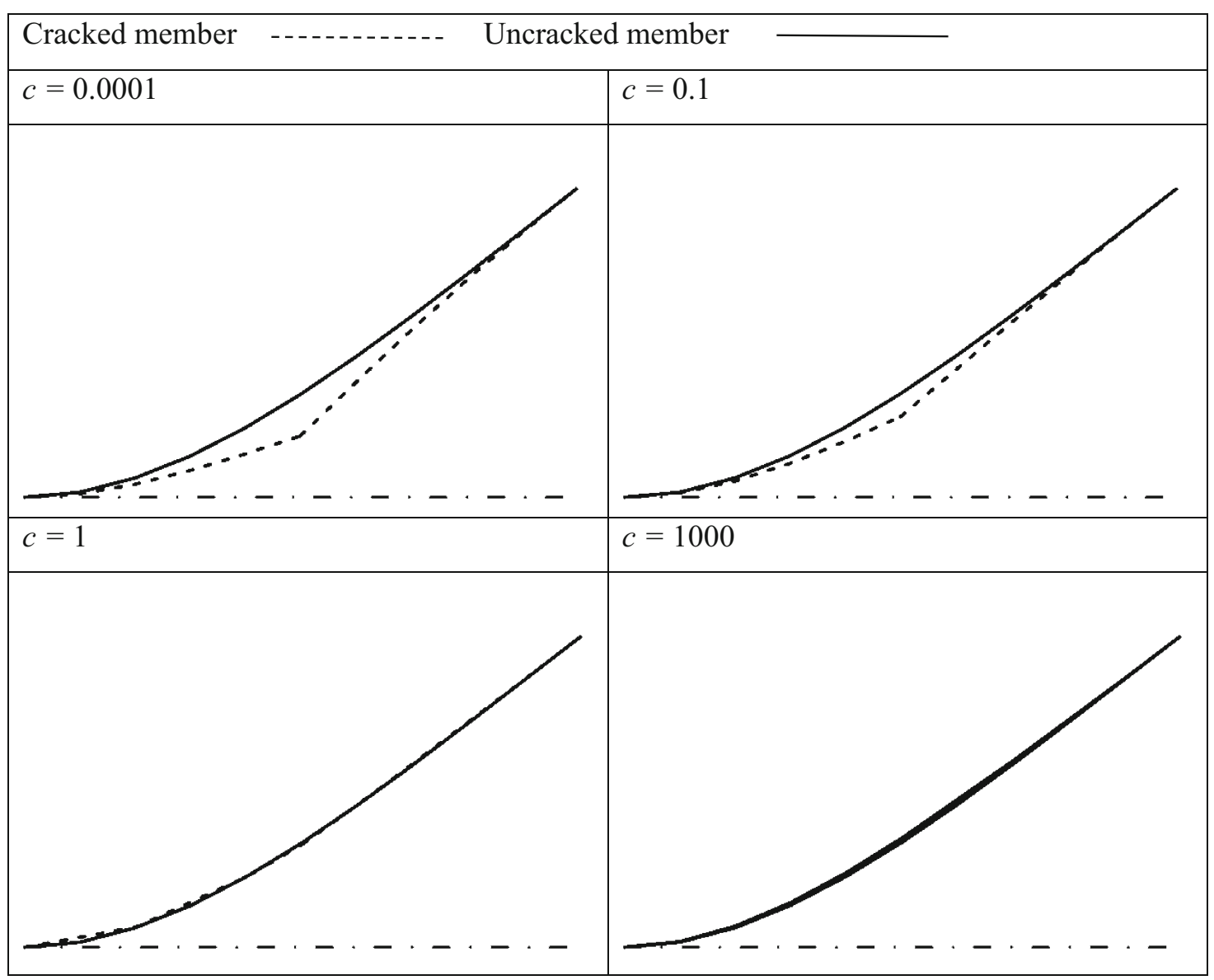

In Table 2 the results of numerical investigations on first shape modes with consideration of different crack size have been shown. The markings of lines are exactly the same as in Table 1 (continuous line-rods 1 and 4; dotted line_-rods 2 and 3). The initiation and propagation of crack have an influence on bent rods axes. An increase in the crack size causes an increase in the area between the axes of rods, which reflects in the reduction in loading capacity and vibration frequency. The greater the crack size, the sharper the connection of rods 2 and 3 .

\subsection{Column $\mathrm{E}_{3}$}

In Figs. 11, 12 and 13 the results of investigations on first vibration frequency have been presented for central crack location and different bending rigidity factor $\mu$ taking into account different crack size for column in $E_{3}$ configuration. The characteristic curves have been plotted in the non-dimensional form. While the very small ( $c=100$ ) crack is taken into account, the highest loading capacity has been observed of each considered bending rigidity factor. The change in $\mu$ from $\mu=0.1$ up to $\mu=1$ results in increase in the bifurcation loading and natural vibration frequency regardless of the crack size. For each considered magnitude of $\mu$ an increase in the crack causes a reduction in the investigated parameters.

In Fig. 14 an influence of the crack size on bifurcation load for three considered magnitudes of bending rigidity factor has been $(\mu=0.1,0.5,1)$ presented. As presented in the Fig. 14 all the curves regardless of bending rigidity factor magnitude are stabilizing. In the $E_{3}$ configuration the high stiffness of the rotational spring is required in order to achieve the final stabilization of bifurcation load magnitude. While the crack size is very big ( $c$ close to zero), the rapid decrease in bifurcation load can be observed. The greatest differences in the magnitude of loading capacity can be observed for $\mu=1$. Fully cracked internal member reflects in 


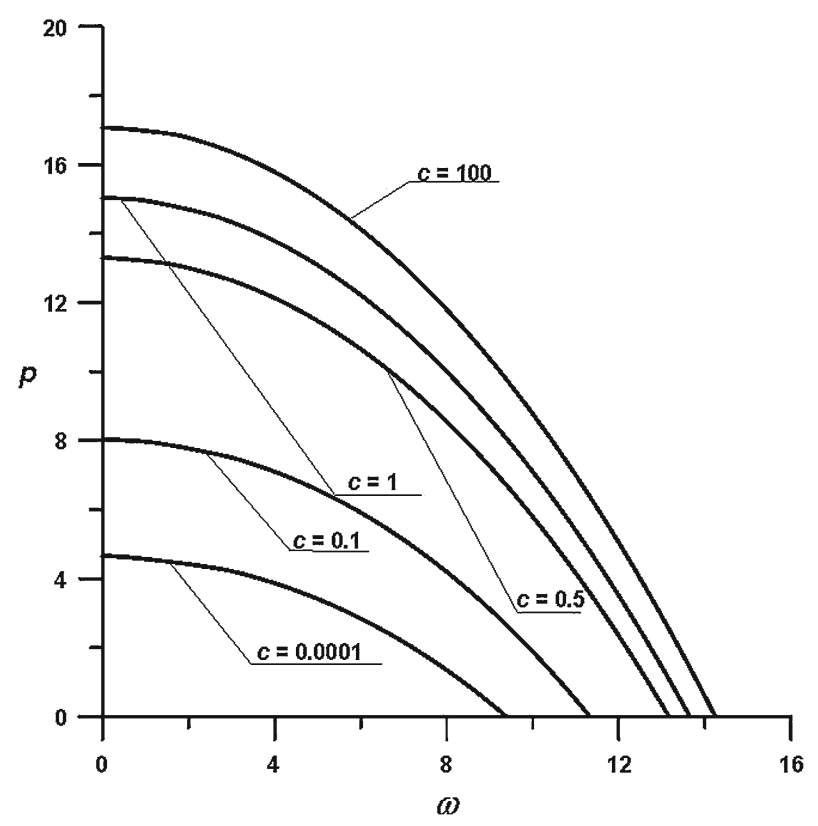

Fig. 11 Characteristic curves on the plane external load-vibration frequency for different crack size, other data: $d_{2}=0.5, \mu=0.1$

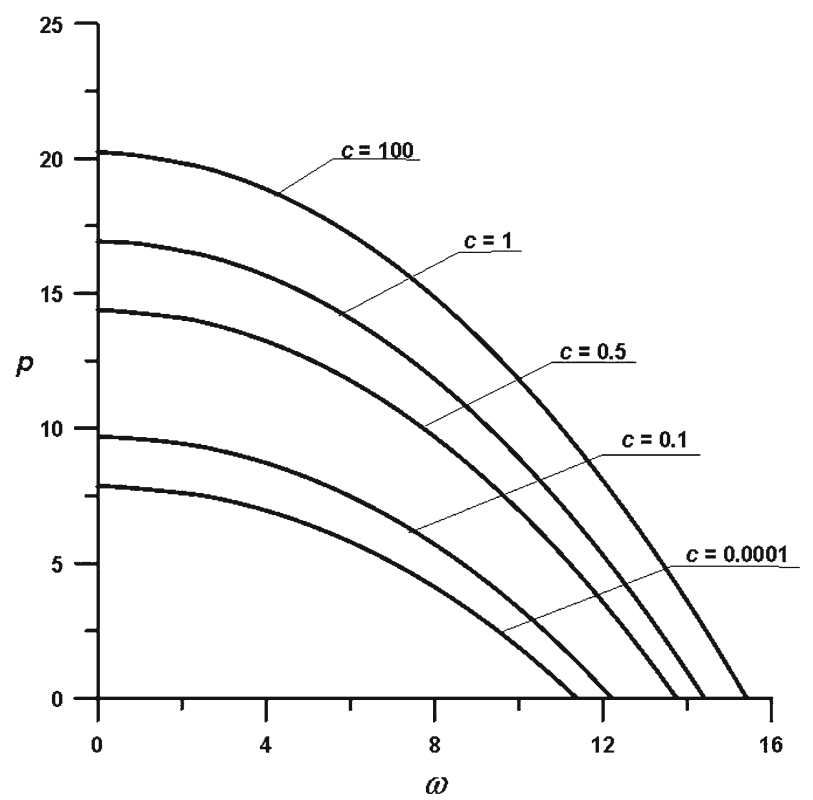

Fig. 12 Characteristic curves on the plane external load-vibration frequency for different crack size, other data: $d_{2}=0.5, \mu=0.5$

the lowest loading capacity. It can be concluded that there is no change in the bifurcation load magnitude for small crack size.

The first shape modes for different crack size have been plotted in Table 3 . The continuous line corresponds to rods 1 and 4 (external elements), while the dotted one stands for rods 2 and 3 (cracked internal member). The vibration frequency and loading capacity highly depend on vibration modes. The greater the difference in the bent rods axes (area between curves), the lower the magnitude of the investigated parameters. T must be stated that location of a crack can be easily observed on the shape modes. 


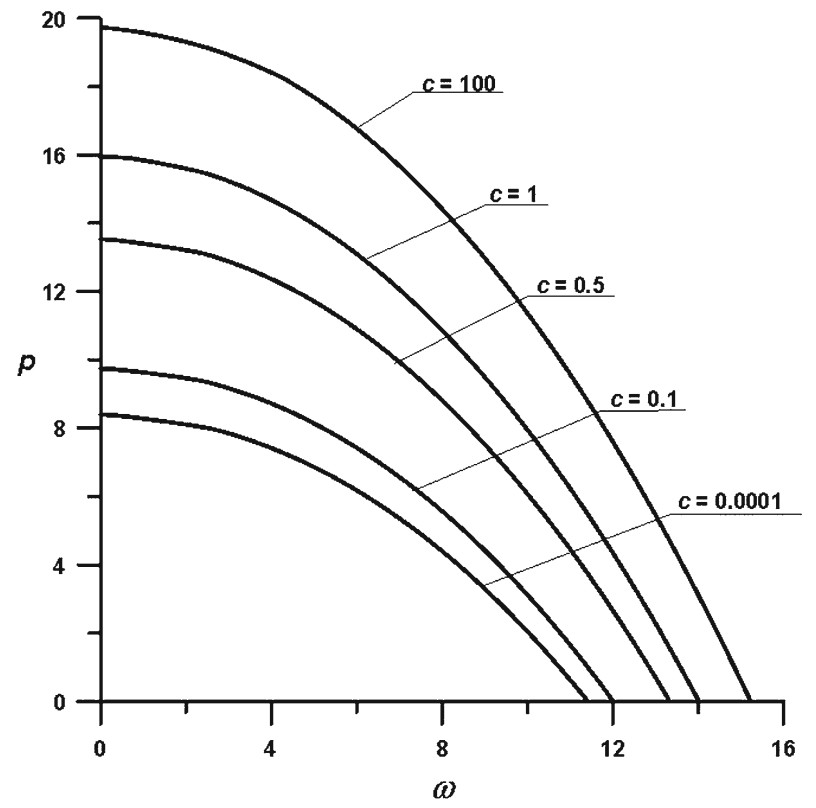

Fig. 13 Characteristic curves on the plane external load-vibration frequency for different crack size, other data: $d_{2}=0.5, \mu=1$

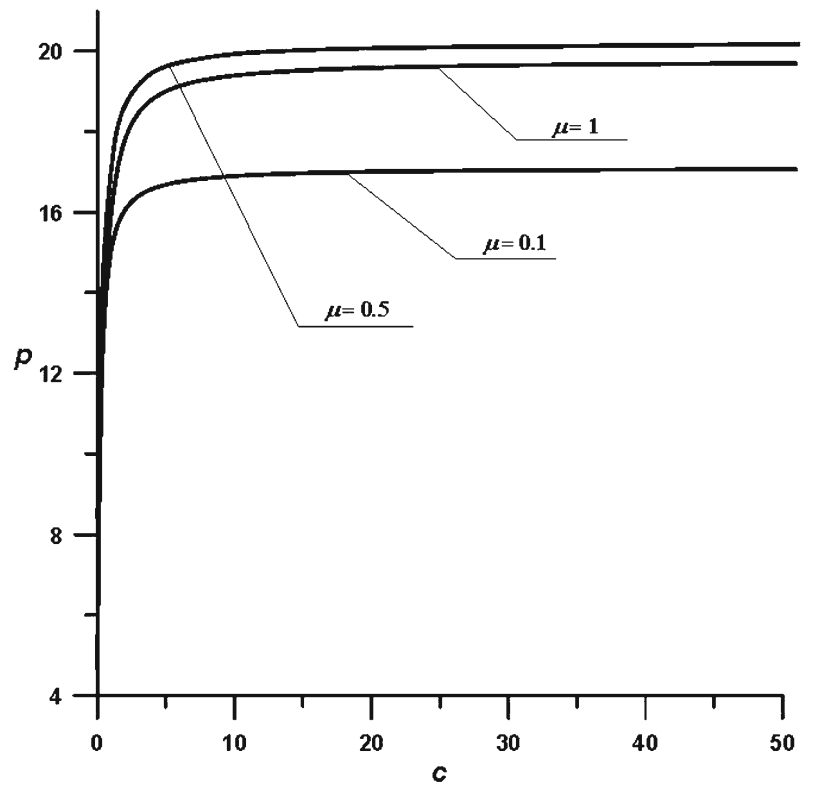

Fig. 14 Curves on the plane external load-crack size for different bending rigidity factor, other data: $d_{2}=0.5$

\subsection{Column $\mathrm{E}_{4}$}

In Figs. 15, 16 and 17 the results of investigations on first vibration frequency have been presented for central crack location and different bending rigidity factor $\mu$ taking into account different crack size for column in $E_{4}$ configuration. The characteristic curves have been plotted in the non-dimensional form. For the very small $(c=100)$ crack the highest loading capacity has been observed regardless of bending rigidity factor magnitude. The change in $\mu$ from $\mu=0.1$ up to $\mu=1$ results in change in natural vibration frequency and bifurcation load. It must be stated that at $\mu=1$ (Fig. 17) there exists such a rotational spring stiffness which simulates crack above which the magnitude of bifurcation load and natural vibration frequency are constant. 
Table 3 Shape modes, $d_{2}=0.5, \mu=0.1$
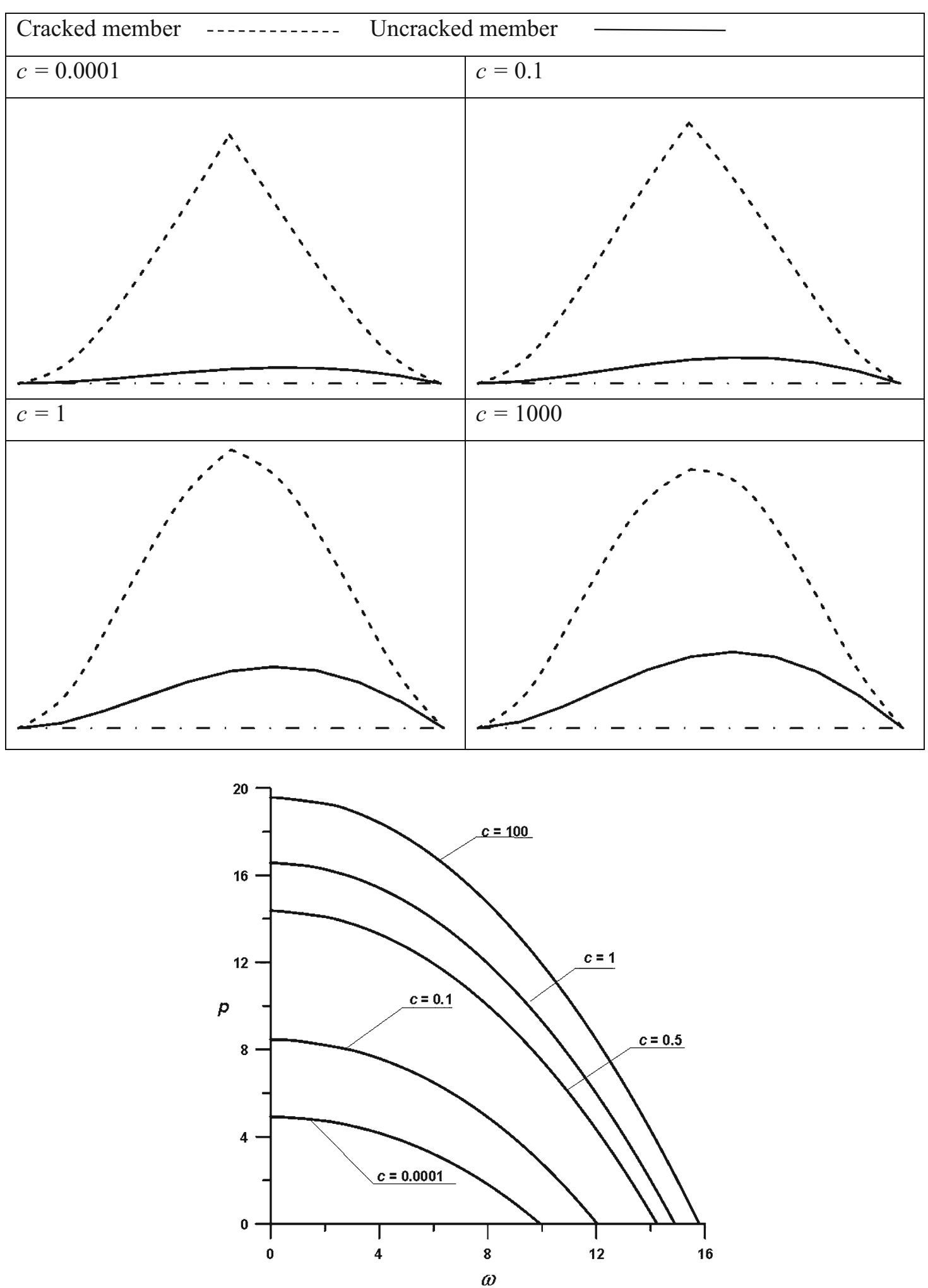

Fig. 15 Characteristic curves on the plane external load-vibration frequency for different crack size, other data: $d_{2}=0.5, \mu=0.1$ 


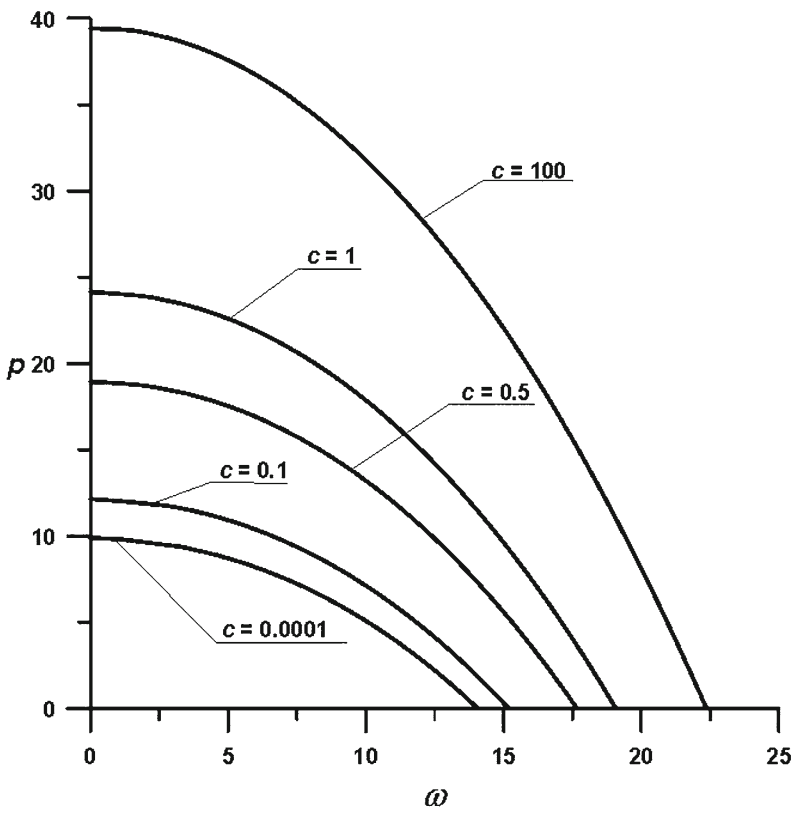

Fig. 16 Characteristic curves on the plane external load-vibration frequency for different crack size, other data: $d_{2}=0.5, \mu=0.5$

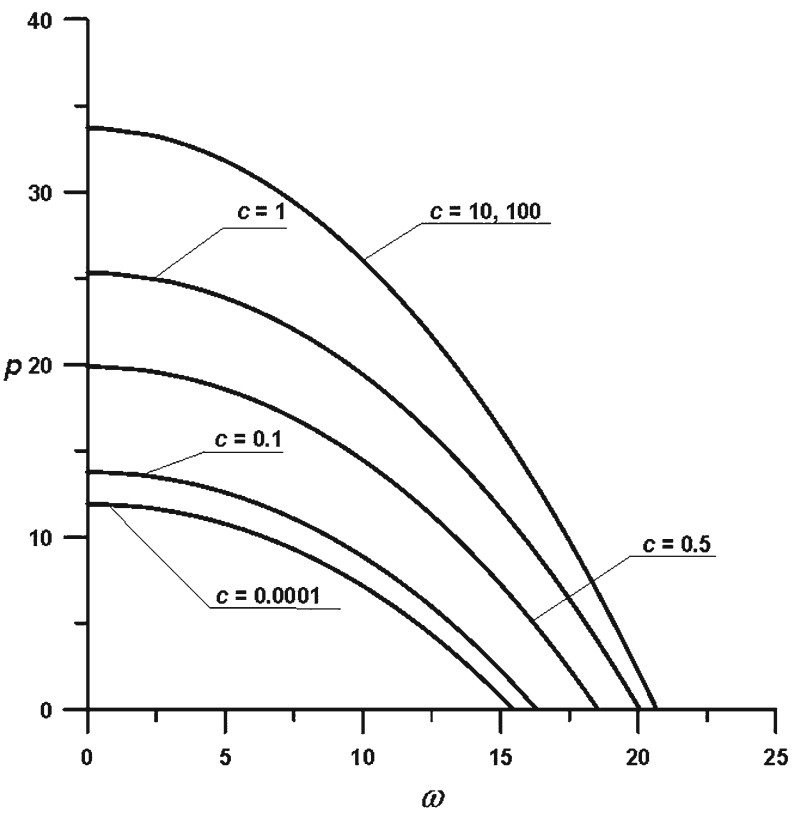

Fig. 17 Characteristic curves on the plane external load-vibration frequency for different crack size, other data: $d_{2}=0.5, \mu=1$

An influence of an increase in the crack results in reduction of magnitudes of investigated parameters regardless of the considered $\mu$, but the size of this reduction depends on $\mu$.

In Fig. 18 an influence of the crack size on bifurcation load for three considered magnitudes of bending rigidity factor $(\mu=0.1,0.5,1)$ has been presented. As shown in Fig. 18, all the curves corresponding to $\mu=0.1,0.5$ are stabilizing with the reduction in the crack size. The lower the magnitude of $\mu$, the faster the stabilization of bifurcation load. For example when $\mu=0.1$ the $c=50$ reflects a uncracked system, while $\mu=0.5$ the $c>50$ is needed to achieve the same situation. In the case when $\mu=1$ is taken into account, the reduction in the crack size causes an increase in the bifurcation load, and at the point marked with black 


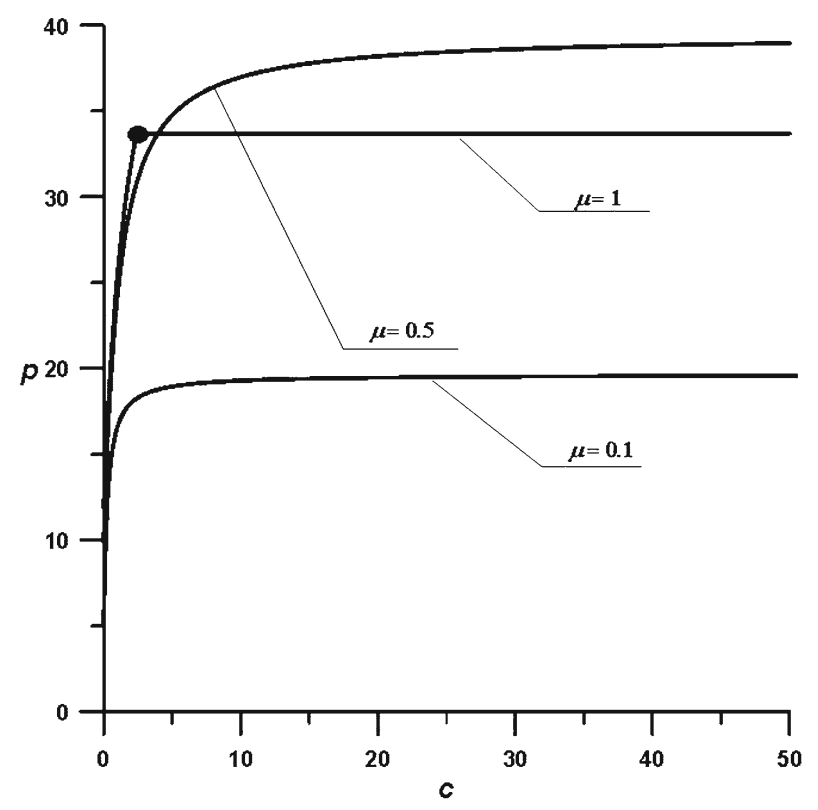

Fig. 18 Curves on the plane external load-crack size for different bending rigidity factor, other data: $d_{2}=0.5$

dot (spring stiffness $c \sim 2.4$ ) the immediate stabilization of the bifurcation load and vibration frequency takes place - see also the characteristic curves in Fig. 17. Furthermore this situation can be also observed on the shape modes. In this case the change in the crack size has no influence of investigated parameters. Once again the fully cracked internal member reflects in the lowest loading capacity.

In Table 4 the first shape modes have been plotted for different crack size. The continuous line corresponds to the external rods ( 1 and 4$)$, while the dotted one stands for cracked internal member. For the crack size $c=0.0001,0.1$ and 1 the internal (cracked) member vibrates, while the external one stays still. An increase in stiffness of the rotational spring causes an external member to vibrate, while an internal one stays still. It can be stated that the vibration modes are now insensitive to spring stiffness.

\subsection{Column $E_{5}$}

In Figs. 19, 20 and 21 the results of investigations on first vibration frequency have been presented for central crack location and different bending rigidity factor $\mu$ taking into account different crack size for column in $E_{5}$ configuration. The characteristic curves have been plotted in the non-dimensional form. In Fig. 19 the curves at bending rigidity factor $\mu=0.01$ are plotted. An increase in the crack size up to $c=0.5$ causes small decrease in bifurcation load and vibration frequency. For greater crack size the decrease in the magnitude of the investigated parameters is greater. When $\mu=0.1$ is taken into account, the bifurcation load and vibration frequency are insensitive to initial grow of $\operatorname{crack}(c=100,1,0.5)$. For greater crack size $(c<0.5)$ the decrease in bifurcation load and vibration frequency can be observed. It is worth noting that these characteristic curves at the beginning are showing high increase in external load with small decrease in the vibration frequency and small increase in external load with high decrease in the vibration frequency at the end. For $\mu=1$ the crack size has no influence on vibration frequency and bifurcation load.

In Fig. 22 an influence of the crack size on bifurcation load for four considered magnitudes of bending rigidity factor $(\mu=0.01,0.1,0.6,1)$ has been presented. The logarithmic scale of the abscissa axis has been used in order to show the most interesting area. As shown in Fig. 22 the curves corresponding to $\mu=0.6,1$ are independent from crack size. At the lower magnitude of $\mu=0.1,0.01$ the great crack size causes rapid decrease in maximum loading capacity. Furthermore the area of independence of loading capacity from crack size can be found for presented $\mu=0.1,0.01$. The described tendency can be also observed in Figs. 19, 20 and 21 after an analysis of the shape of characteristic curves. Additionally an analysis of the shape modes has been done. 
Table 4 Shape modes, $d_{2}=0.5, \mu=1$
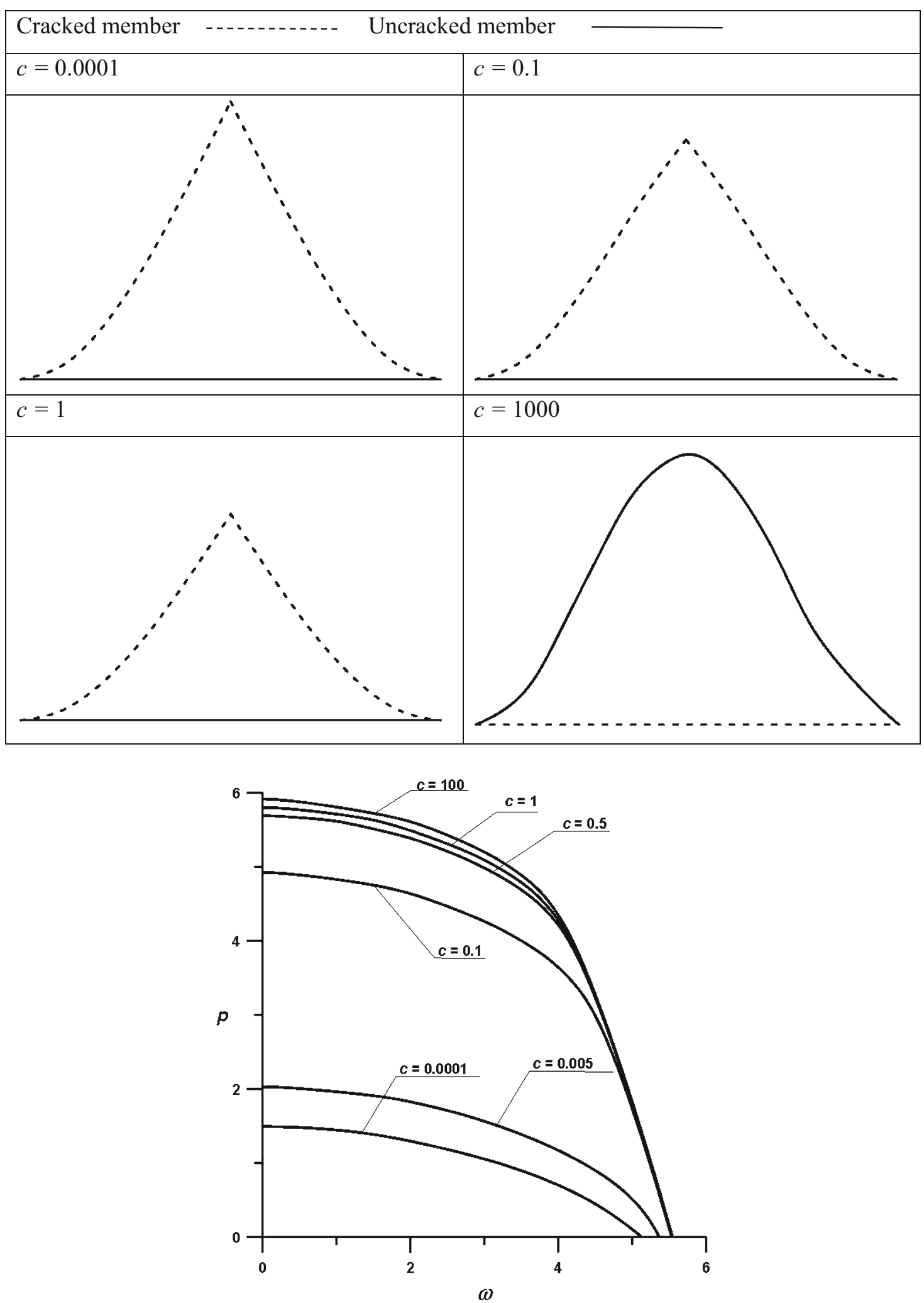

Fig. 19 Characteristic curves on the plane external load-vibration frequency for different crack size, other data: $d_{2}=0.5, \mu=$ 0.01 


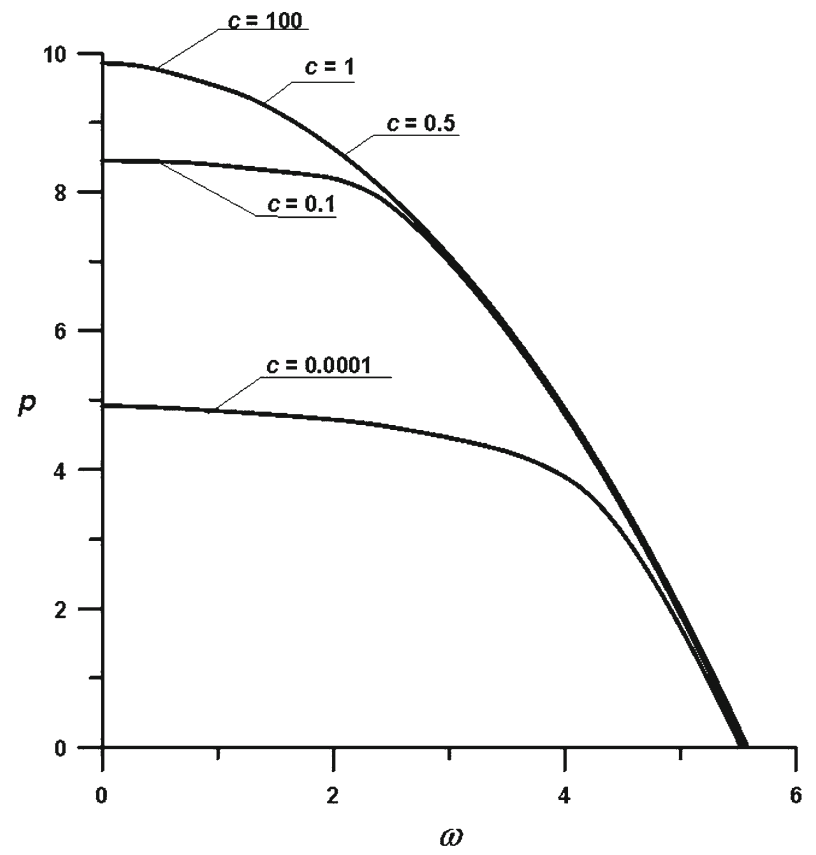

Fig. 20 Characteristic curves on the plane external load-vibration frequency for different crack size, other data: $d_{2}=0.5, \mu=0.1$

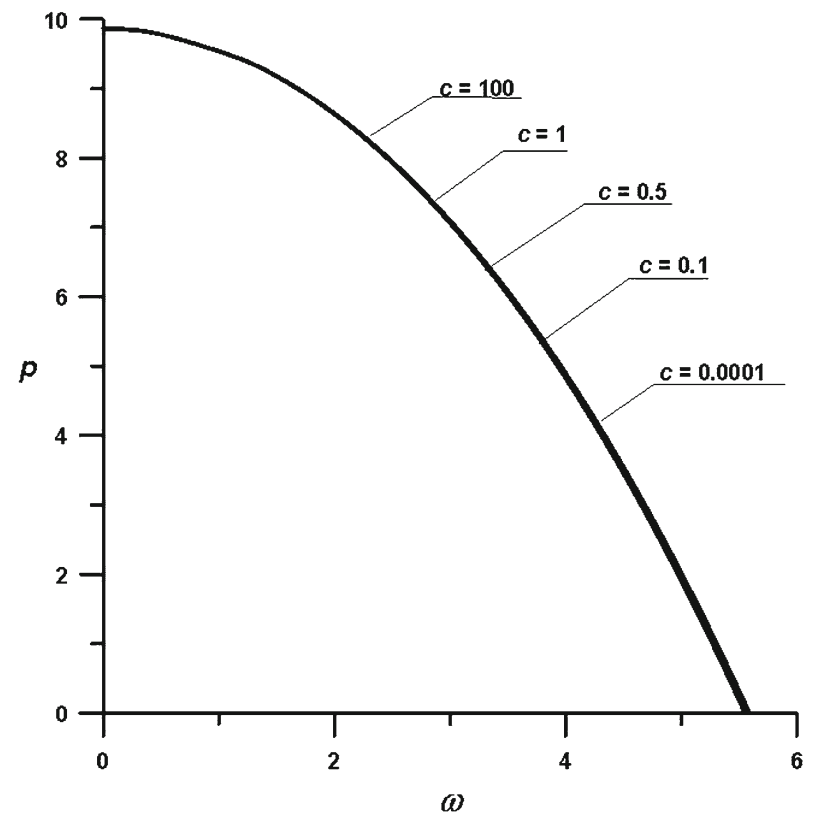

Fig. 21 Characteristic curves on the plane external load-vibration frequency for different crack size, other data: $d_{2}=0.5, \mu=1$

In Tables 5 and 6 the first shape modes have been plotted for different crack size and bending rigidity factor $(\mu=0.01$ and 1$)$ with reference to Figs. 19, 20 and 21. The continuous line corresponds to the external rods ( 1 and 4$)$, while the dotted one stands for cracked internal member. When the results presented in Table 5 are investigated, it can be noticed that the change in crack size highly affects the shape modes as well as characteristic curves. The greatest area between the axes of rods was found at $c=0.0001$. For higher spring stiffness, the area is reduced up to constant value at $c>1$. The results gathered in Table 6 refer to the characteristic curves plotted in Fig. 21. As shown a change in the crack size has negligibly small influence on 


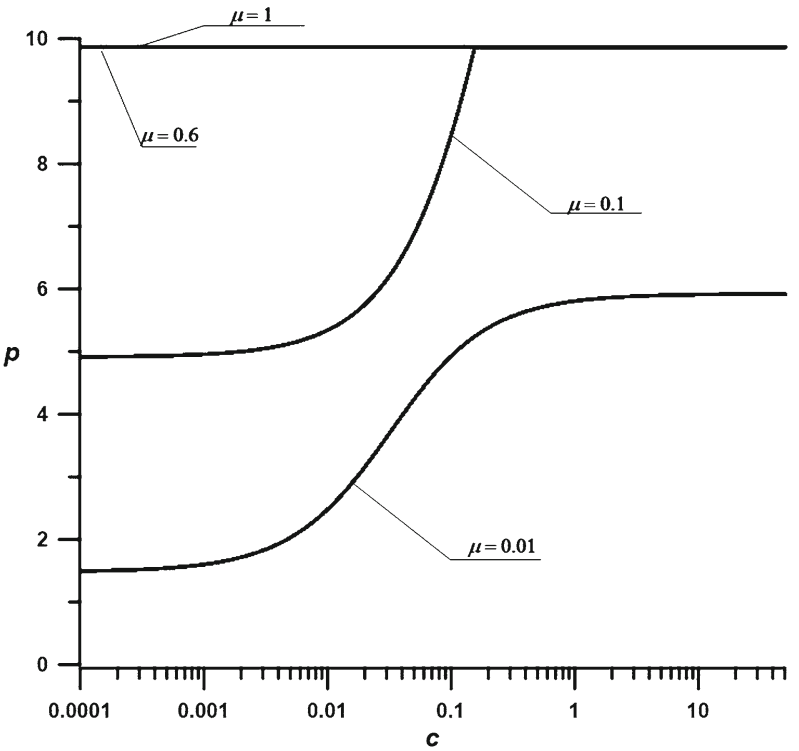

Fig. 22 Characteristic curves on the plane external load-crack size for different bending rigidity factor, other data: $d_{2}=0.5$

Table 5 Shape modes, $d_{2}=0.5, \mu=0.01$

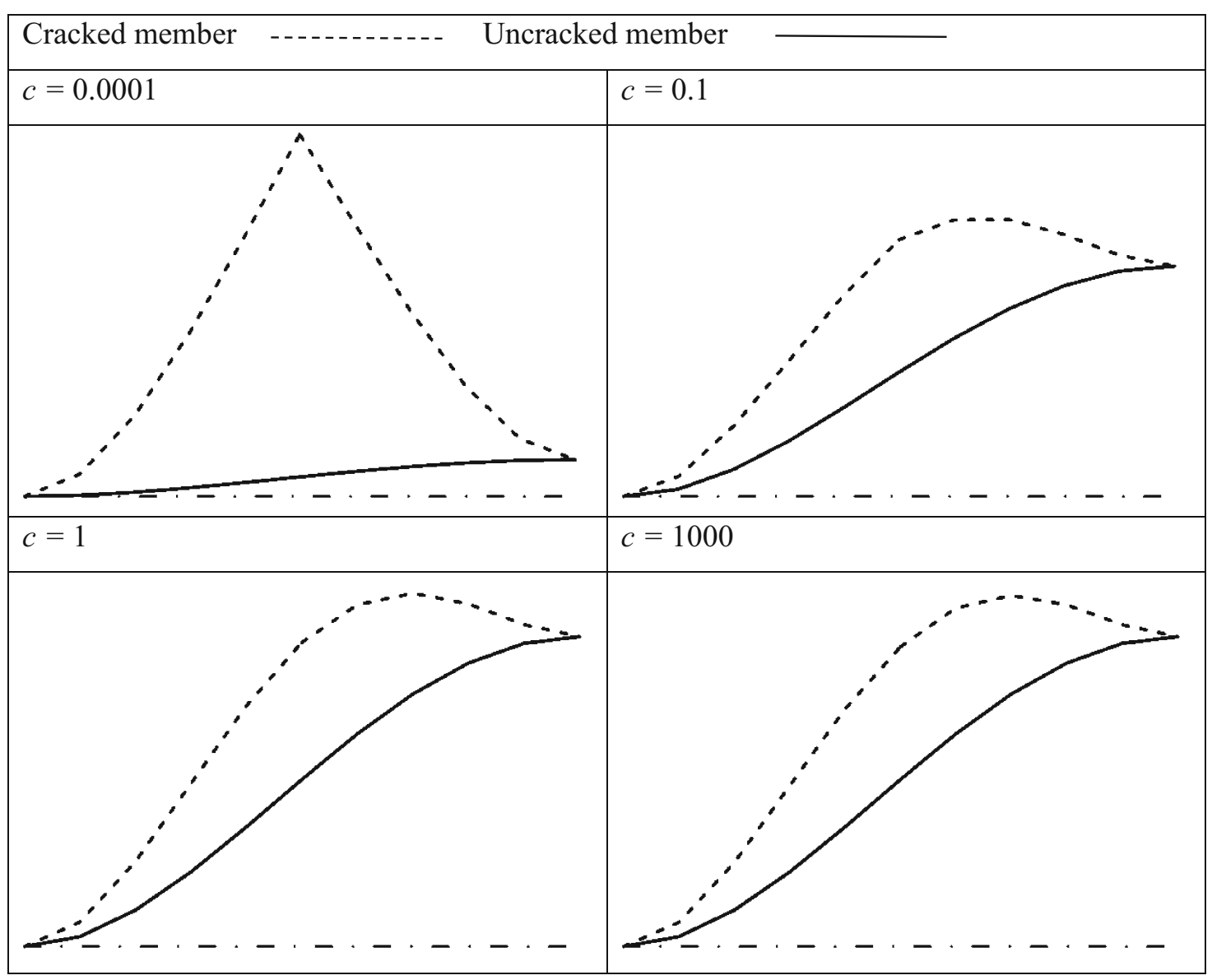


Table 6 Shape modes, $d_{2}=0.5, \mu=1$

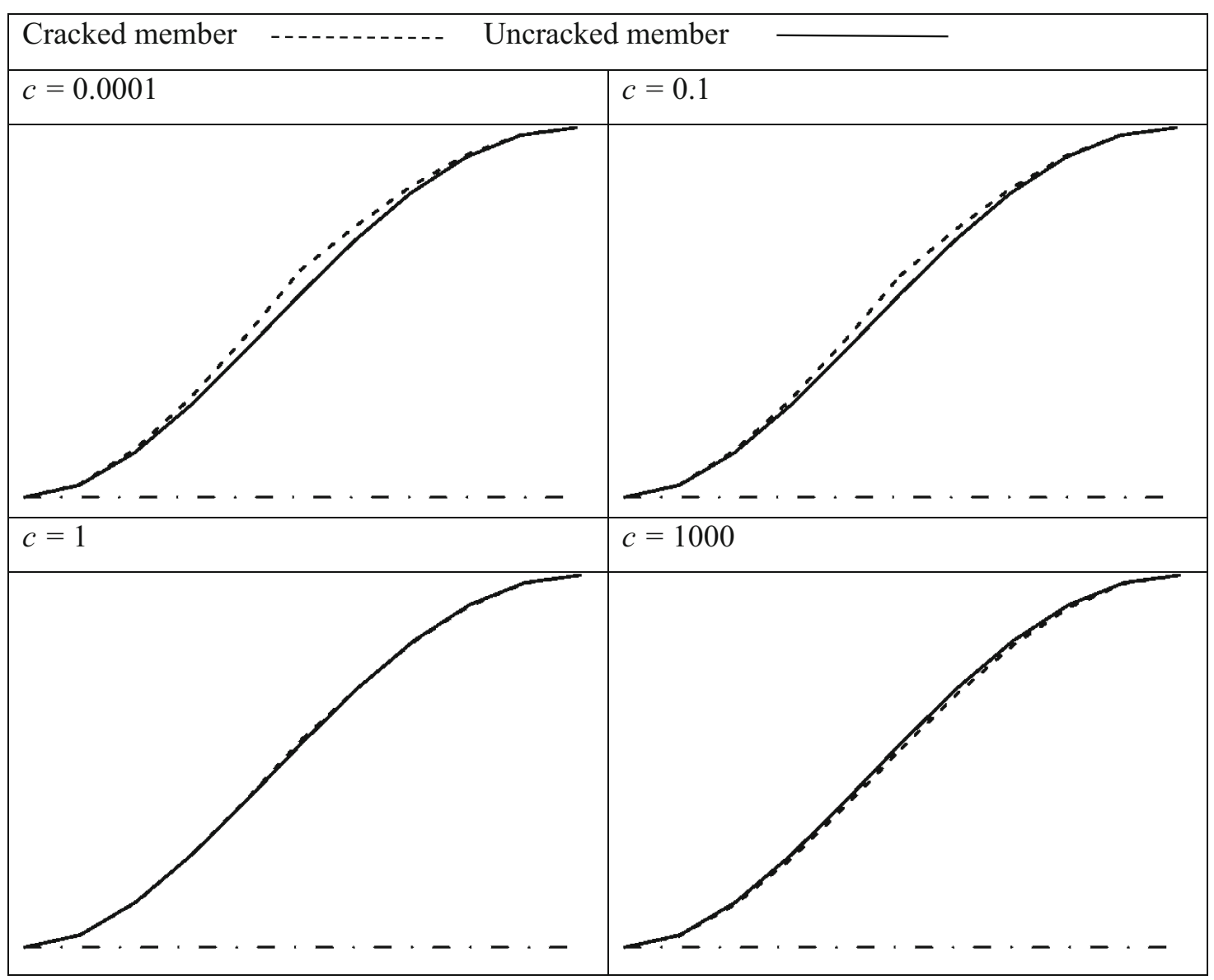

the shape modes. It can be concluded that such a magnitude of bending rigidity factor can be found at which the shape of characteristic curves (and shape modes) are independent from crack size.

\section{Conclusion}

In this paper an influence of the crack size on instability and natural vibrations of multi-member columns has been presented. The obtained results of numerical simulations can be used to monitor the dynamic behavior of the nonlinear slender systems. In the manuscript five different configurations of the multi-member columns are shown. On the basis of smart mathematical model the simulation of columns with different boundary conditions can be easily achieved. On the basis of the above investigations the following conclusion can be done:

- if the stiffness of the rotational spring which simulates the crack tends to infinity, the magnitude of the bifurcation load and vibration frequency can be treated as ones computed for corresponding linear system of uncracked multi-member one.

- the crack size has a great influence on the bifurcation load magnitude and vibration frequency. An increase in the crack size affects the characteristic curves causing the reduction in the investigated parameters. The size of the this reduction depends on chosen initial configuration of the column as well as bending rigidity factor.

- there can be found such a bending rigidity factor magnitude at which an influence of the crack size is negligible. 
- for each configuration of the investigated slender system the proper combination of bending rigidities of rods allows one to achieve required dynamic characteristic. Which is being disrupted by presence of the crack.

- in the case when the crack affects the characteristic curves, the lowest magnitudes of investigated parameters have been found.

- the crack size has an influence on the shape modes. At low spring stiffness the transversal displacement of the cracked member is the greatest. Also it can be observed that the change in the crack size changes the shape modes (vibrating / still member).

- on the basis of the presented sample of the results of numerical simulations the monitoring of the dynamic behavior can be done in order to find the crack initiation.

- at some magnitudes of the bending rigidity factor this monitoring can be problematic due to small or negligible influence of the crack on the characteristic curves/shape modes. That is why the additional monitoring systems must be used.

In this paper the linear component of vibration frequency around the rectilinear form of static equilibrium is presented. The further investigation can consider the nonlinear component of natural vibrations which depends on amplitude of vibration and shape modes. The next interesting step in the analysis of the cracked multi-member slender systems may concern on curvilinear form of static equilibrium. The rationale of such investigation is that the bifurcation load magnitude at which the change in equilibrium form takes place highly depends on crack size and location (see Figs. 6,10, 14, 18, 22). Therefore in some cases the system can be characterized by the presence of a curvilinear form of static equilibrium in higher range of external load magnitudes than at rectilinear one.

Open Access This article is distributed under the terms of the Creative Commons Attribution 4.0 International License (http:// creativecommons.org/licenses/by/4.0/), which permits unrestricted use, distribution, and reproduction in any medium, provided you give appropriate credit to the original author(s) and the source, provide a link to the Creative Commons license, and indicate if changes were made.

\section{References}

1. Andersen, S.B., Thomsen, J.J.: Post-critical behavior of Beck's column with a tip mass. Int. J. Non Linear Mech. 37, 135-151 (2002)

2. Anifantis, N., Dimarogonas, A.: Stability of columns with a single crack subjected to follower and axial loads. Int. J. Solids Struct. 19, 281-291 (1981)

3. Arif Gurel, M.: Buckling of slender prismatic circular columns weakened by multiple edge cracks. Acta Mech. 188, 1-19 (2007)

4. Batabyal, A.K., Sankar, P., Paul, T.K.: Crack detection in cantilever beam using vibration response. Springer Proc. Phys. 126, 27-33 (2009)

5. Beck, M.: Die Kincklast des einsetig eingespanuten trangential gedruck Stabes. Zeit. Ang. Math. Phys. 3, 225-228 (1952)

6. Binici, B.: Vibration of beams with multiple open cracks subjected to axial force. J. Sound Vib. 287, 277-295 (2005)

7. Chati, M., Rand, R., Mukherjee, S.: Modal analysis of a cracked beam. J. Sound Vib. 207(2), 249-270 (1996)

8. Chinchalkar, S.: Determination of crack location in beams using natural frequencies. J. Sound Vib. 247(3), 417-429 (2001)

9. Chondros, T.G., Dimarogonas, A.D., Yao, J.: Vibration of a beam with a breathing crack. J. Sound Vib. 239, 57-67 (2001)

10. Chondros, T.G.: The continuous crack flexibility model for crack identification. Fatigue Fract. Eng. Mater. Struct. 24, 643-650 (2001)

11. Chondros, T.G., Dimarogonas, T.G.: Dynamic sensitivity of structures to cracks. J. Vib. Acoust. Stress Reliab. Des. 111, 251-256 (1989)

12. Chondros, T.G., Dimarogonas, T.G., Yao, J.: A continuous cracked beam vibration theory. J. Sound Vib. 215, 17-34 (1998)

13. Evensen, D.A.: Nonlinear vibrations of beams with various boundary conditions. AIAA 6, 370-372 (1968)

14. Kukla, S.: Free vibrations and stability of stepped columns with cracks. J. Sound Vib. 319, 1301-1311 (2009)

15. Lee, J., Bergman, L.A.: The vibration of stepped beams and rectangular plates by an elemental dynamic flexibility method. J. Sound Vib. 171, 617-640 (1994)

16. Leung, A.Y.T.: Exact spectral elements for follower tension buckling by power series. J. Sound Vib. 309, 718-729 (2008)

17. Li, Q.S.: Classes of exact solutions for buckling of multi-step non-uniform columns with an arbitrary number of cracks subjected to concentrated and distributed axial loads. Int. J. Eng. Sci. 41(6), 569-586 (2003)

18. Nandwana, B.P., Maiti, S.K.: Detection of the location and size of a crack in stepped cantilever beams based on measurements of natural frequencies. J. Sound Vib. 203(3), 435-466 (1997)

19. Osiński, Z.: Vibration Theory. PWN, Warszawa (1978)

20. Przybylski, J.: Influence of the supporting spring stiffness on the vibrations and stability of a geometrically nonlinear column. J. Theor. Appl. Mech. 39(1), 129-152 (2001) 
21. Przybylski, J., Tomski, L., Gołębiowska-Rozanow, M., Szmidla, J.: Vibration and stability of columns subjected to a certain type of generalised load. J. Theor. Appl. Mech. 37(2), 283-289 (1999)

22. Peng, Z.K., Lang, Z.Q., Billings, S.A.: Crack detection using nonlinear output frequency response functions. J. Sound Vib. 301(1), 777-788 (2007)

23. Qian, G.L., Gu, S.N., Jiang, J.S.: The dynamic behavior and crack detection of a beam with a crack. J. Sound Vib. 225(1), 201-208 (1990)

24. Roodr, J., Chilver, A.H.: Frame buckling an illustration of the perturbation technique. Int. J. Non Linear Mech. 8, 237-255 (1970)

25. Sokół, K.: The local and global instability and vibration of a nonlinear column subjected to Euler's load. Sci. Res. Inst. Math. Comput. Sci. Czestochowa Univ. Technol. 1, 187-194 (2010)

26. Sokół, K.: Linear and nonlinear vibrations of a column with an internal crack. J. Eng. Mech. 140(5), 04014021 (2014)

27. Tomski, L.: Vibration and Stability of Slender Systems. WNT, Warszawa (2004)

28. Tomski, L., Uzny, S.: Vibration and stability of geometrically nonlinear column subjected to generalized load with a force directed toward the positive pole. Int. J. Struct. Stab. Dyn. 8(1), 1-24 (2008a)

29. Tomski, L., Uzny, S.: Free vibration and the stability of a geometrically non-linear column loaded by a follower force directed towards the positive pole. Int. J. Solids Struct. 45(1), 87-112 (2008b)

30. Tomski, L., Szmidla, J.: Local and global instability and vibration of overbraced Euler's column. J. Theor. Appl. Mech. 41(1), 137-154 (2003)

31. Uzny, S.: Free vibrations of an elastically supported geometrically nonlinear column subjected to a generalized load with a force directed toward the positive pole. J. Eng. Mech. ASCE 137(11), 740-748 (2011) 